University of Louisville

ThinkIR: The University of Louisville's Institutional Repository

Electronic Theses and Dissertations

8-2012

\title{
Idiopathic pulmonary fibrosis : diagnosis, management, and the search for a cure.
}

Daniel Fioret

University of Louisville

Follow this and additional works at: https://ir.library.louisville.edu/etd

\section{Recommended Citation}

Fioret, Daniel, "Idiopathic pulmonary fibrosis : diagnosis, management, and the search for a cure." (2012). Electronic Theses and Dissertations. Paper 437.

https://doi.org/10.18297/etd/437

This Master's Thesis is brought to you for free and open access by ThinkIR: The University of Louisville's Institutional Repository. It has been accepted for inclusion in Electronic Theses and Dissertations by an authorized administrator of ThinkIR: The University of Louisville's Institutional Repository. This title appears here courtesy of the author, who has retained all other copyrights. For more information, please contact thinkir@louisville.edu. 


\title{
IDIOPATHIC PULMONARY FIBROSIS: \\ DIAGNOSIS, MANAGEMENT, AND THE SEARCH FOR A CURE
}

\author{
By \\ Daniel Fioret \\ B.A., Centre College, 2010
}

\begin{abstract}
A Thesis
Submitted to the Faculty of the

School of Medicine of the University of Louisville

in Partial Fulfillment of the Requirements

for the Degree of
\end{abstract}

Master of Science

Department of Pharmacology and Toxicology

University of Louisville

Louisville, Kentucky

August, 2012 


\title{
IDIOPATHIC PULMONARY FIBROSIS:
}

\section{DIAGNOSIS, MANAGEMENT, AND THE SEARCH FOR A CURE}

\author{
By \\ Daniel Fioret \\ B.A., Centre College, 2010
}

A Thesis Approved on

August 2, 2012

by the following Thesis Committee:

Jesse Roman M.D.

Gavin E. Arteel Ph.D.

J. Christopher States Ph.D. 


\section{ACKNOWLEDGEMENTS}

I would like to thank my mentor, Dr. Roman, for his guidance and patience during the past two years. His leadership was paramount in the success of my research and reaching my full potential. I would also like to thank the other committee members Drs. Arteel and States for their assistance and valuable feedback. For all of my efforts in the

laboratory, I would like to thank the other members of Dr. Roman's laboratory including Jeff Ritzenthaler, Edilson Torres, Glenn Vicary, Wenjing Zhang, Yu Fan, and Caleb Greenwell. Also, I would like to thank my family for their continued support. 


\author{
ABSTRACT \\ IDIOPATHIC PULMONARY FIBROSIS: \\ DIAGNOSIS, MANAGEMENT, AND THE SEARCH FOR A CURE \\ Daniel Fioret
}

August 2, 2012

Idiopathic pulmonary fibrosis (IPF) is a fibrotic lung disease with poor prognosis. To address this disease broadly, the candidate engaged in clinical work, literature analysis, database analysis, and laboratory work. The clinical work culminated in a case report that highlights the controversies inherent in the diagnosis and treatment of IPF. The literature analysis discusses ongoing clinical trials and challenges inherent in the management of IPF. Evaluation of a national database unveiled information about the burden of IPF on the healthcare system. Finally, the laboratory work examined the role of oxidant stress and the interaction of lung fibroblasts and lung cancer cells. Altogether, the document defines the clinical presentation of IPF, summarizes current treatment strategies and obstacles to the development of effective therapies, describes the burden of IPF to healthcare regarding hospitalization rates and costs, and beings to elucidate mechanisms by which IPF patients are more susceptible to lung cancer. 


\section{TABLE OF CONTENTS}

PAGE

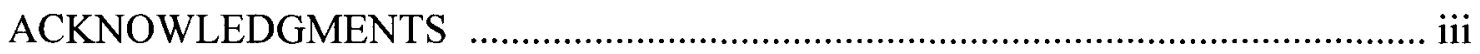

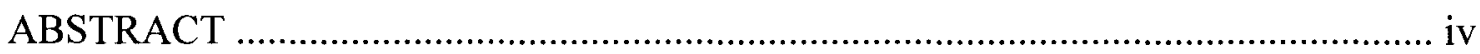

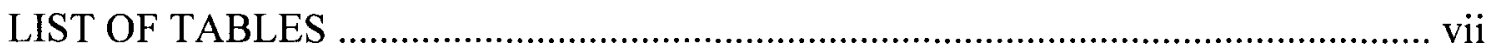

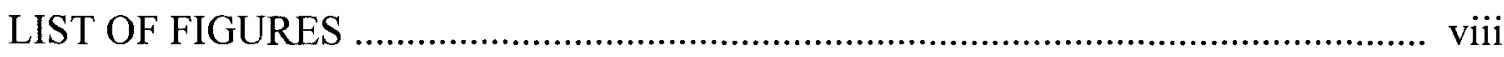

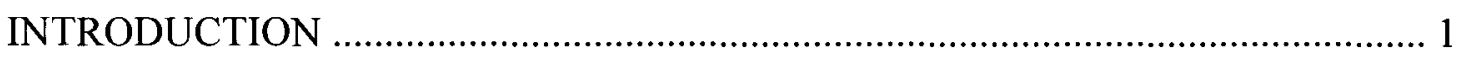

MATERIALS AND METHODS …………………........................................ 3

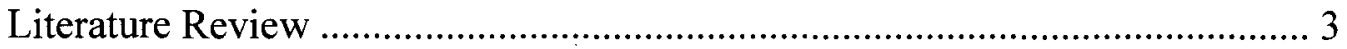

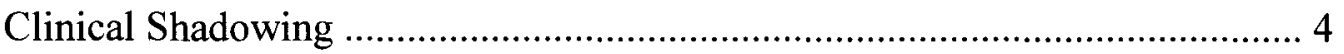

Preparation of Clinical Manuscript ................................................................... 4

Analysis of National Database .................................................................... 5

Investigation of Fibrosis Through Laboratory Work ........................................ 5

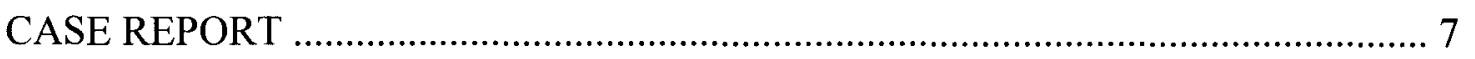

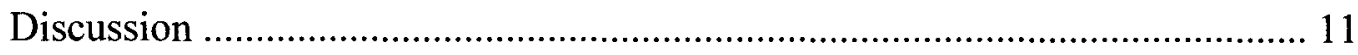

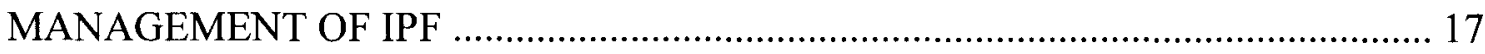

The Search for Effective Therapies in IPF ....................................................... 18

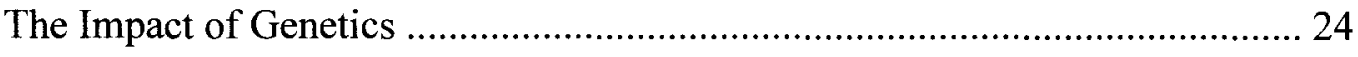

Other Important Considerations in IPF ………………………………….... 25

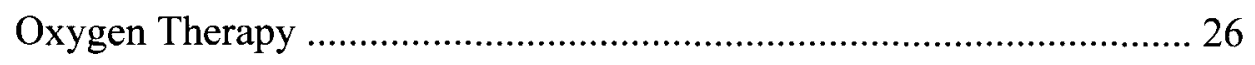

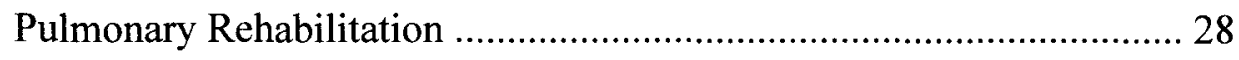

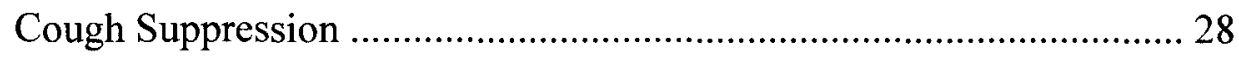

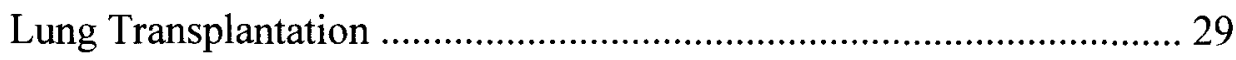

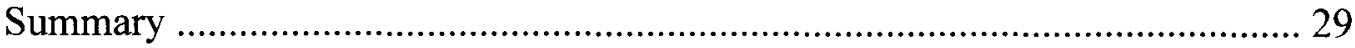


PAGE

IMPACT TO HEALTHCARE - EPIDEMIOLOGICAL STUDY

Methods .......................................................................................... 32

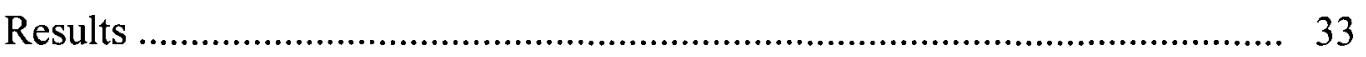

Discussion .................................................................................. 57

IDENTIFYING TARGETS FOR INTERVENTION (LABORATORY WORK) .... 64

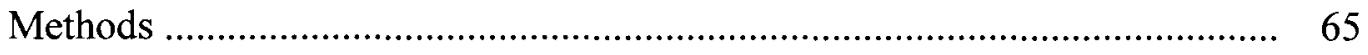

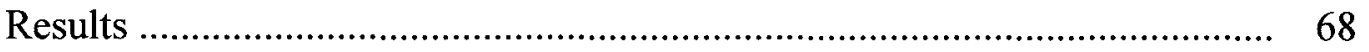

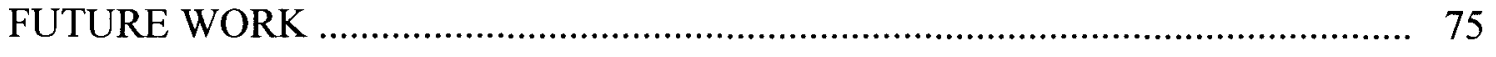

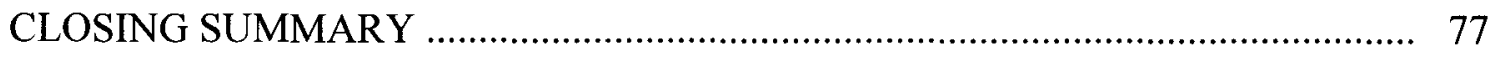

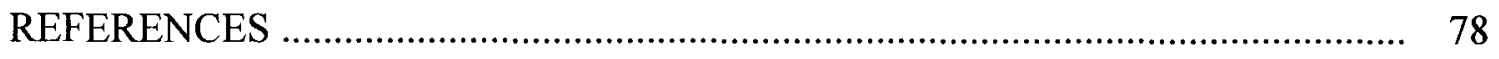

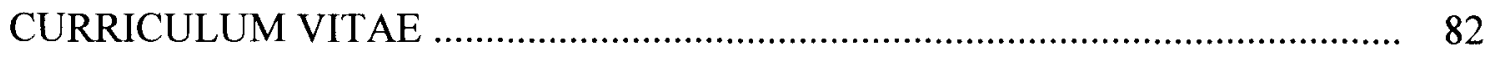




\section{LIST OF TABLES}

$\underline{\text { TABLE }}$

$\underline{\text { PAGE }}$

1. Drugs found to be ineffective in the treatment of IPF.

2. Drugs requiring further investigation in the treatment of IPF. 20

3. Drugs considered having some efficacy but require further testing. 21

4. Hospitalization charges, LOS, and mortality for patients hospitalized for IFA, PPF, and sarcoidosis (2008)

5. Primary procedures for patients hospitalized with IFA, PPF, and sarcoidosis (2009)

6. Secondary diagnoses for patients hospitalized with IFA, PPF or sarcoidosis (2009)

7. Primary diagnoses for patients hospitalized with secondary diagnoses of IFA, PPF, or sarcoidosis (2009)

\section{ABBREVATIONS USED:}

1. IPF - Idiopathic pulmonary fibrosis

2. LOS - Length of stay

3. IFA - Idiopathic fibrosing alveolitis

4. PPF - Postinflammatory pulmonary fibrosis 


\section{LIST OF FIGURES}

\section{FIGURE}

$\underline{\text { PAGE }}$

1. High-resolution computer tomography (HRCT) in a patient with IPF.

2. Evaluation schematic for patients suspected of interstitial lung disease (ILD)

3. General management of patients with IPF.

4. Hospital discharges. A, Hospital discharges for all conditions.

B, Hospital discharges for PPF, IFA, and sarcoidosis.

5. Hospital discharges separated by all-listed and principal diagnoses.
A, Hospital discharges for IFA. B, Hospital discharges for PPF.

$C$, Hospital discharges for sarcoidosis.

6. Length of stay by condition and region. A, Length of stay for PPF, IFA, sarcoidosis and all conditions. B, Length of stay for IFA based on region. C, Length of stay for PPF based on region. $D$, Length of stay for sarcoidosis based on region.

7. Length of stay by payer. A, Length of stay for IFA.

B, Length of stay for PPF. C, Length of stay for sarcoidosis.

8. Length of stay by gender. A, Length of stay for IFA.

B, Length of stay for PPF. C, Length of stay for sarcoidosis.

9. Discharges by gender. A, Length of stay for IFA.

$B$, Length of stay for PPF. C, Length of stay for sarcoidosis.

$\mathrm{D}$, Length of stay for all conditions.

10. Discharge location. A, Percent discharged home.

$\mathrm{B}$, Percent discharged to home health care.

C, Percent discharged to another short-term hospital.

$\mathrm{D}$, Percent discharged to another institution (nursing home, rehab).

11. In-hospital mortality by disease and gender.
A, In-hospital mortality for IFA, PPF, Sarcoidosis, and all conditions.
B, In-hospital mortality for IFA. C, In-hospital mortality for PPF.
$\mathrm{D}$, In-hospital mortality for sarcoidosis.

12. In-hospital mortality by age group. A, In-hospital mortality for IFA.

B, In-hospital mortality for PPF.

13. Mean charges by condition and region. A, Mean hospital

charges by IFA, PPF, sarcoidosis, and all conditions.

B, IFA. C, PPF. D, Sarcoidosis. 
14. Mean charges by payer. A, IFA. B, PPF. C, Sarcoidosis. 52

15. Mean charges by gender. A, IFA. B, PPF. C, Sarcoidosis. 54

16. Effect of income on mortality. A, IFA. B, PPF.

17. Discharges by age group. A, All conditions. B, IFA.

C, PPF. D, Sarcoidosis. $\quad 56$

18. Discharges/million people by increasing average state age for 2010 .

A, IFA. B, PPF. C, Sarcoidosis. $\quad 58$

19. Lewis lung carcinoma (LLC) cell viability assay - response to redox media. A, 24 hour response. B, 48 hour response. C, 72 hour response. 69

20. H460 and H1792 human cancer cells cell viability assay - response to redox media. A, 24 hour response in $\mathrm{H} 460$ cells. B, 24 hour response in $\mathrm{H} 1792$ cells. C, 48 hour response in $\mathrm{H} 460$ cells.

$\mathrm{D}, 48$ hour response in $\mathrm{H} 1792$ cells.

21. H460 and H1792 human cancer cells - response to redox media (continued). A, 72 hour response in $\mathrm{H} 460$ cells.

B, 72 hour response in $\mathrm{H} 1792$ cells. 72

22. Caspase assay in LLC cells (72 hour response). 74

\section{ABBREVATIONS USED:}

1. IPF - Idiopathic pulmonary fibrosis

2. LOS - Length of stay

3. IFA - Idiopathic fibrosing alveolitis

4. PPF - Postinflammatory pulmonary fibrosis 


\section{IDIOPATHIC PULMONARY FIBROSIS: DIAGNOSIS, PATHOGENESIS AND THE SEARCH FOR A CURE}

Idiopathic pulmonary fibrosis (IPF) is a disease with high mortality and of unknown etiology. Research efforts attempting to elucidate the mechanisms behind the development of this lethal fibrotic disease have yet to determine a definitive mechanism. It is likely that there are multiple mechanisms by which the disease can progress, which may explain the fact that no single intervention has been proven effective. Therefore, although researchers and clinicians have been working on this disease for several decades and this has resulted in improved classification and identification of IPF patients, clinical outcomes remain unchanged.

It is evident that a narrow approach to furthering knowledge about IPF is not sufficient. Furthering understanding of this disease by multiple strategies will result in improved outcomes for these patients faster than by one method alone. We wanted to learn more about the presentation and management of these patients as well as the impact of the disease to healthcare in the U.S. We hypothesized that IPF hospitalization rates and costs were increasing and, if so, this should serve as a justification for further efforts to address this devastating disease. To test our hypothesis, a multi-step approach was used. This document analyzes the disease from the standpoint of the patient, and then expands into current management/treatment options, followed by an analysis of the 
burden of the disease using a national hospital database. It concludes with an investigation of the cellular effects of oxidant stress, a mechanism implicated in the pathogenesis of IPF and its consequences. Altogether, this thesis helps to improve knowledge about identification, clinical management, healthcare burden/hospitalization statistics and disease pathogenesis.

\section{INTRODUCTION}

IPF is a chronic and progressive pulmonary disease characterized by progressive deterioration of respiratory function due to lung fibrosis. More than 150,000 Americans are affected by IPF, which is the most common and severe form of the idiopathic interstitial pneumonias [1]. The progressive fibrosis typical of IPF results in dyspnea that ultimately causes to respiratory failure (and death) within 3 to 5 years of diagnosis in patients with advanced disease $[2,3]$. The situation is further complicated, since there are no FDA approved drugs to treat IPF. Lung transplantation and oxygen supplementation are the only treatment options that have been shown to benefit patients with IPF.

Because of its progressive nature and the lack of effective therapies, an early and accurate diagnosis of IPF is crucial. A typical patient with IPF is older than 50 years and bibasilar crackles can be heard during the physical examination. Imaging studies reveal bilateral infiltrates with peripheral and basilar predominance, traction bronchiectasis and honeycombing. Physiological abnormalities with a restrictive pattern are common and often associated with hypoxemia at rest or during exertion. Lung histology reveals heterogeneous distribution of lung fibrosis, honeycombing, fibroblastic foci and a paucity of inflammation; this pattern is known as a pattern of usual interstitial pneumonitis (UIP) [4]. 
A diagnosis of IPF is difficult, because the clinical manifestations are similar to other conditions (e.g., sarcoidosis) and is in part a diagnosis of exclusion [5]. Other conditions that resemble IPF are interstitial lung diseases related to connective tissue disorders such as rheumatoid arthritis, systemic lupus erythematosus and progressive systemic sclerosis, and conditions triggered by environmental exposures, such as chronic hypersensitivity pneumonitis $[6,7]$. An accurate diagnosis of IPF is important considering the differences in prognosis, IPF shows only a $\sim 50 \% 3$-year survival rate. More importantly, no therapies have been proven to reverse, halt or delay the progression of disease in IPF in large, well-conducted, double-blinded, placebo-controlled, prospective clinical trials $[3,8]$.

Diagnosing IPF requires three main criteria: 1, The exclusion of other known sources of interstitial lung diseases (including occupational, environmental, and drug toxicities obtained through a thorough patient history); 2, A pattern of usual interstitial pneumonia on highresolution computed tomography (HRCT) (if a surgical lung biopsy is not available/safe to obtain); 3, Specific combinations of patterns from HRCT and surgical lung biopsy when both tests are available. Diagnostic accuracy can be further improved with multidisciplinary talks between specialists [3].

Herein, we describe our attempts to understand IPF through the description of a case and a summary of the treatment/management options currently available to these patients, the obstacles inherent in developing safe and effective therapies. Afterwards, we discuss the burden of the disease focusing on hospitalization rates and costs in the U.S. We end by an exploration of the role of oxidant stress in disease pathogenesis. 


\section{METHODS}

The data prepared for this thesis were generated through independent but complementary activities: 1) Review of current literature, 2) Exposure to IPF patients at the clinic, 3) Preparation of a manuscript that describes an IPF patient's presentation, 4) Preparation of a manuscript that summarizes the current understanding of IPF treatment, 5) Analysis of a national database to determine the impact of IPF on hospitalization costs, and 6) Investigation of fibrosis through laboratory work. This work was furthered through interactions with Dr. Roman and his colleagues at the clinic as well as in the laboratory. The experience culminated with the presentation of this work at several local and international venues and the publication of manuscripts in the area of IPF ( 2 published, 1 submitted, 1 in preparation).

\section{Literature Review}

My initial introduction to the fibrotic lung disease known as IPF began at the start of my projects with Dr. Roman. I initially devoted my efforts to researching the available literature related to IPF. I focused my search through PubMed and began looking at review articles and currently published papers related to IPF. In addition, Dr. Roman helped to focus my search with suggested articles to read. This led to a better understanding of the disease and also complimented the time I spent shadowing Dr. Roman at his pulmonary clinic.

\section{Clinical Shadowing}

The opportunity to add to my literary studies with direct patient interaction was as rewarding as it was informative. I joined Dr. Roman at his clinic once a week and was able to 
watch his interaction with his patients, several of whom had IPF. Dr. Roman was an excellent teacher and helped me to better understand the role of a physician in the management of a chronic deadly disease. One of the patients that I had a chance to meet served as an example of an IPF patient for the clinical case report I helped write.

\section{Preparation of Clinical Manuscript [9]}

To prepare the clinical manuscript describing a patient with IPF, I utilized the physician notes and laboratory write-ups about the patient. I summarized the relevant information as well as adding relevant introductory and discussion sections to complement the clinical data.

\section{Preparation on Manuscript of IPF Management [10]}

My efforts studying the available literature also allowed me to write an article on the management of IPF in the clinical arena. The article summarized the current treatment options and strategies for IPF along with an update about the available drugs being tested for the treatment of IPF.

\section{Analysis of National Database to Determine the Impact of IPF on Hospitalizations}

\section{[11]}

To address trends related to IPF on a broad scale, I utilized a hospital database known as the Healthcare Cost and Utilization Project Network (HCUPnet) database. Data available 
through the HCUPnet Database provide access to national hospitalization data in the U.S. between 1993 and 2008. We analyzed data related to ICD-9-CM codes for post inflammatory pulmonary fibrosis (PPF, ICD-9 code 515) and idiopathic fibrosing alveolitis (IFA, ICD-9 code 516.3) and compared them to those of sarcoidosis (ICD-9 code 135).

\section{Investigation of Fibrosis through Laboratory Work}

To help address the lack of understanding of disease pathogenesis, I also devoted time to laboratory research. My efforts focused on working with mouse and human fibroblasts with an "IPF phenotype". We received several human fibroblast cell lines from patients with IPF. We found it difficult to work with cell lines from the human patients perhaps due to cell aging. The cells appeared to be stuck in a state of relative senescence and only some of the cell lines would grow at any noticeable rate. As a result of this, my work centered on recreating the conditions of an IPF phenotype in mouse fibroblasts focusing on oxidant stress.

\section{6.a. Specific laboratory methods}

The laboratory experiments necessary for this study involved cell culture, proliferation assays, Western blot, and an apoptosis assay. Detailed methods for each of these techniques are described in the section about laboratory work towards identifying targets for intervention later in this paper. 


\section{BODY OF THESIS}

\section{CASE REPORT}

[9] - Fioret, D., et al., A case of progressive lung fibrosis. Am J Med Sci, 2011. 341(6): p. 428-30.

A 73-year-old obese white man with a history of high blood pressure came to the hospital after noticing a lump on his chest. A biopsy of the lump proved it to be harmless, but the chest radiograph revealed lung abnormalities that prompted a chest tomogram and further evaluation. Ultimately, a video-assisted thoracoscopy-guided lung biopsy was performed that revealed alternating areas of relatively normal lung mixed with a patchwork of parenchymal tissue involvement by end-stage honeycombing. Areas of active fibrosis with fibroblastic foci and collagen deposition were also seen in addition to mild inflammation consistent with a pattern of usual interstitial pneumonia. The patient was given a diagnosis of IPF and sent to the University of Louisville Interstitial Lung Disease Center for further management.

On evaluation, the patient complained of dyspnea on exertion that had been worsening over the past 2 years. Mild cough was present, but no other symptoms suggestive of infection or heart disease were elicited. He had a history of tobacco use that involved smoking 6 to 8 cigarettes a day for more than 30 years; he quit smoking 22 years ago and was not drinking alcohol or taking alternative medicine therapies. During questioning about occupational and environmental exposures, the patient acknowledged working on a farm and being exposed to hay, cattle and other animals on a regular basis 
throughout his life. Prescribed medications included fluticasone and salmeterol inhalation once daily, a tablet combining valsartan and hydrochlorothiazide for hypertension, diltiazem, Tums, and omeprazole for acid indigestion and gastro-esophageal reflux disease, pentosan polysulfate sodium, acetylsalicylic acid, fish oil and a multivitamin.

The initial physical examination showed normal vital signs with a hemoglobin oxygen saturation at room air and at rest value of $94 \%$ and a respiratory rate of 14 breaths/min. His head examination revealed no lesions, but he had a slightly narrowed oropharynx. Examination of the neck revealed no jugular venous distention, lymphadenopathy or thyromegaly. His heart rate was regular, but showed a systolic $2 / 6$ murmur that was most noticeable in the left sternal border. The lung examination revealed bibasilar inspiratory crackles but no ronchi or wheezes. The abdominal examination was benign. Extremities showed no edema, and his fingers showed borderline clubbing. His electrocardiogram revealed a heart rate of 61 beats/min but was otherwise normal. A right and left heart catheterization performed by his cardiologist for atypical chest pain revealed mild disease in the right coronary artery and the circumflex artery, as well as moderate disease in the mid left anterior descending coronary artery. The study also revealed evidence of very mild pulmonary hypertension.

During his initial visit, pulmonary function tests revealed no obstruction, but the total lung capacity was decreased $(4.19 \mathrm{~L})$, and the diffusion lung capacity was severely reduced (10.04 40\% predicted). These results were interpreted as consistent with a severe restrictive ventilator pattern and severe reduction in diffusing capacity. More importantly, the results were dramatically decreased from those obtained 4 years earlier which showed a total lung capacity of $5.2 \mathrm{~L}$ ( $78 \%$ predicted) and a normal diffusion lung 
capacity $\left(24.7,119 \%\right.$ predicted). The patient's blood gas showed a $\mathrm{pH}$ of $7.429, \mathrm{Pco}_{2}$ of 37.4 and $\mathrm{a} \mathrm{Po}_{2}$ of 65 . A 6-minute walk test revealed mild hemoglobin oxygen desaturation to $90 \%$. A chest tomogram revealed fibrotic lung changes with peripheral distribution and basilar predominance, honeycombing and traction bronchiectasis.

After evaluation of his overall clinical picture, imaging studies and histology, a diagnosis of IPF was confirmed. Treatment options were discussed, and the patient opted for participating in a placebo-controlled randomized clinical trial. A few weeks after inclusion in the trial, the patient developed swelling and a white exudate over his tongue, considered to be candidiasis, for which he was treated with fluconazole. The exudates cleared, but the patient had 2 episodes of worsening shortness of breath for which his primary physician gave him intramuscular steroid injections.

Approximately 2 months after inclusion in the trial, the patient developed significant shortness of breath on exertion that was persistent and associated with increased nonproductive cough and significant fatigue. Pulmonary function tests revealed further deterioration. During a 6-minute walk test, his hemoglobin oxygen saturation (initially at $93 \%$ ) dropped to $71 \%$ within 2 minutes. Because of the possibility of an "exacerbation of IPF," the patient was admitted to the hospital where he underwent an extensive evaluation. A chest tomogram using a protocol to detect pulmonary embolism revealed the earlier reported changes of fibrosis but no new infiltrates, ground glass opacification, pleural effusion or other abnormalities, including pulmonary embolism (Figure 1). An echocardiogram failed to reveal a cardiac etiology for his deterioration and sputum, and blood cultures showed no organisms. A bronchoscopy revealed significant airway erythema and some yellowish secretions. The patient was 


\section{Figure 1}

\section{High-resolution computer tomography (HRCT) in a patient with IPF}

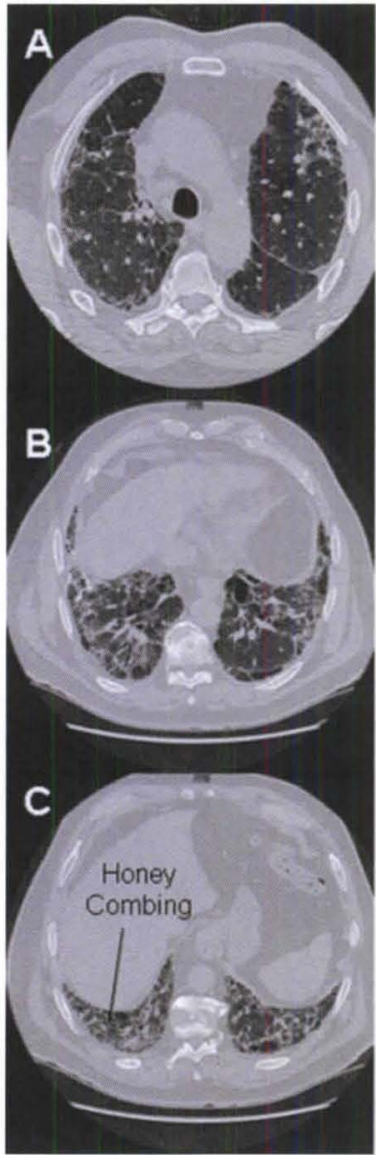

Peripheral

Distribution

\section{Basilar}

Predominance

In idiopathic pulmonary fibrosis HRCT typically shows bilateral infiltrates predominating in the periphery (image A), limited disease in the upper lobes (image B) and worsening infiltrates toward the lung bases (C). Fibrosis is noted mainly at the bases where honeycombing is observed (C). 
given a diagnosis of severe acute bronchitis and was started on antibiotics and intravenous steroids. After 3 days hospitalization, the patient was discharged home on supplemental oxygen and oral antibiotics to be followed in clinic. After initial improvement, the patient has continued to deteriorate with worsening dyspnea on exertion and has increased requirements for oxygen supplementation.

\section{DISCUSSION}

IPF has received a great deal of attention lately due to the recognition that this deadly illness affects more than 150,000 Americans, that its incidence is increasing, [1] and by the increased number of clinical trials available to patients. Despite the above, this case highlights many of the difficulties inherent in the diagnosis and management of patients with IPF. Often, and despite increased awareness about the disease, patients with IPF have symptoms for more than 2 years before a diagnosis is made. Diagnosis is delayed because of the slow insidious course of the disease in an aging population (IPF occurs mainly in subjects older than 50 years) that often has an increased frequency of exercise-limiting conditions, such as heart disease [12].

An early diagnosis of IPF is important, given differences in prognosis and in management with earlier detection. The 3-year survival rate for IPF is $\sim 50 \%$, and it has no cure, whereas survival is better in other idiopathic interstitial pneumonias and related interstitial disorders [2]-[3]. Also, there is no effective therapy for IPF, whereas immunosuppression may be effective in other conditions associated with lung inflammation and fibrosis [12]-[13]. 
An accurate diagnosis of IPF requires a thorough patient history and physical examination, and a careful analysis of diagnostic examinations and imaging studies. The patient history should include job history, smoking and drinking habits, associated diseases, family medical history and any exposure to environmental or occupational irritants such as metal dusts, paints and animals/pets. The latter is especially important as these exposures may cause conditions that could be confused with IPF such as chronic hypersensitivity pneumonitis, a disorder included in the differential diagnosis of our case [3].

A thorough evaluation for autoimmune causes of pulmonary fibrosis that includes scleroderma, rheumatoid arthritis and systemic lupus erythematosus should be undertaken. The clinical history is most critical here, and a screening antinuclear antibody and rheumatoid factor serology, among other serologies, should be obtained [3].

The physical examination is not particularly revealing, except for the presence of bibasilar crackles, which sometimes leads to the erroneous diagnosis of congestive heart failure [14]. Digital clubbing is relatively frequent $(25-50 \%$ of patients) but is not specific to, or necessary for, the diagnosis of IPF [12]. Pulmonary function tests usually reveal a restrictive pattern and marked decrease in gas transfer as measured by the diffusing capacity for carbon monoxide. Hemoglobin oxygen saturation should be evaluated both at rest and exertion as abnormalities may only be detected during exertion during the early stages of the disease.

Chest radiography is useful, but it may overlook significant changes. It typically shows bilateral reticular infiltrates (typically peripheral and basilar) and small lung 
volumes. Upper lobe predominance or the presence of other findings such as significant lymphadenopathy, pleural effusions and/or calcifications and cardiomegaly should point to alternative diagnoses. A high-resolution chest tomogram (HRCT) is crucial for the evaluation of subjects with interstitial lung disease [3]. In IPF, typical chest tomography findings include bilateral peripheral infiltrates with basilar predominance associated with traction bronchiectasis and, often, honeycombing. In the right clinical setting, and if the fibrosis is considered idiopathic, a chest tomogram with the above findings is sufficient to confirm the diagnosis of IPF [15]. In the absence of the typical findings on HRCT, and especially if ground glass opacification is present, a surgical biopsy might be necessary for establishing the diagnosis. However, the benefits of a surgical biopsy must be weighed against the risk of the procedure, and the age of this population and accompanying medical conditions such as heart disease, hypertension and diabetes must be taken into consideration.

Bronchoscopy, bronchoalveolar lavage and transbronchial lung biopsy are helpful to rule out infection and granulomatous disorders (e.g., sarcoidosis) but rarely provide sufficient material to evaluate histological patterns that is crucial for an accurate diagnosis of the idiopathic interstitial pneumonias. It is for this reason that a surgical biopsy is often considered for a definitive diagnosis [16-18]. As in our patient, histology shows a pattern of usual interstitial pneumonitis, which is characterized by heterogeneous distribution of fibrosis, paucity of inflammation, the presence of fibroblastic foci and honeycombing. However, this histological pattern is not entirely specific and may be elusive even in the hands of the most experienced pathologists [19]. Figure 2 


\section{Figure 2}

Evaluation schematic for patients suspected of interstitial lung disease (ILD)

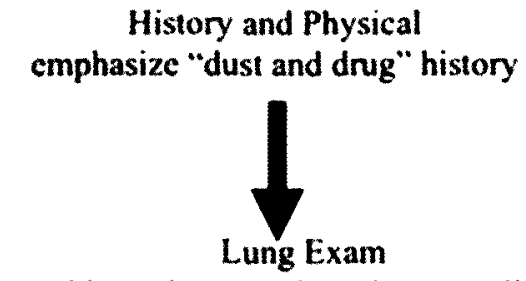

crackles; stigmata of autoimmune disease

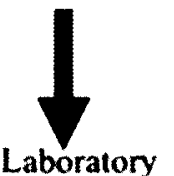

Comp panel: CBC with diff: ANA. RF, other serologies

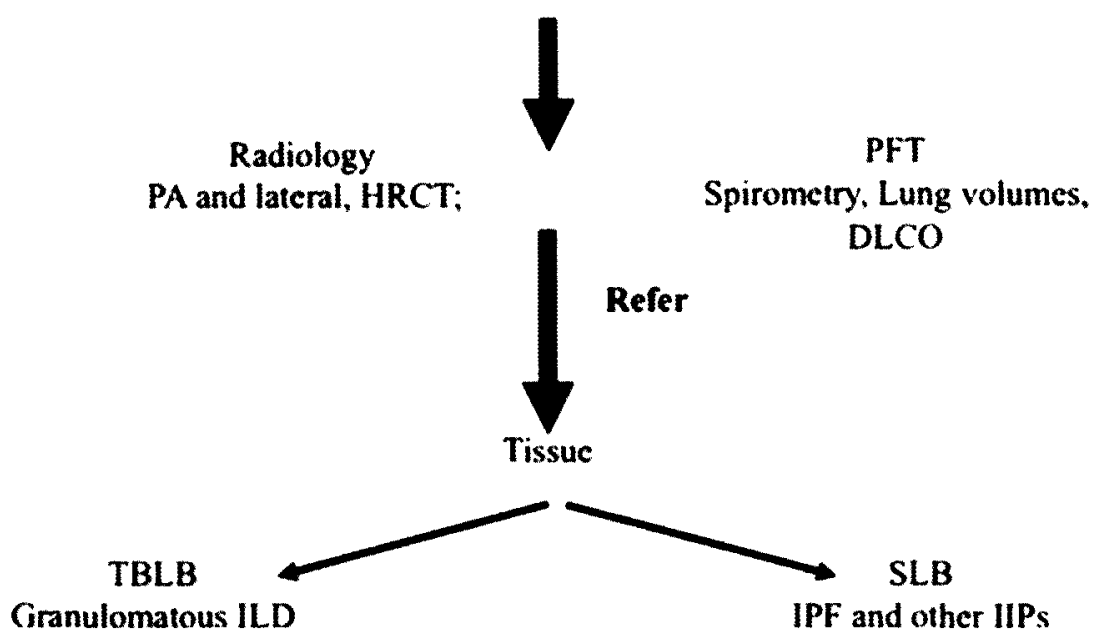

The pulmonary history is paramount in the evaluation of ILD. Initial laboratory work should include a complete metabolic profile (Comp panel) and antinuclear antibody (ANA) and rheumatoid factor (RF) screen. A postero-anterior (PA) and lateral chest film will define the diffuse bilateral abnormalities typical of the ILD, and the high-resolution computerized tomogram (HRCT) adds specificity and can resolve abnormalities not seen on plain film. When tissue is needed for diagnosis and management decisions, the patient is referred for biopsy. Transbronchial lung biopsy (TBLB) is the procedure of choice when there is suspicion of granulomatous disorder such as sarcoidosis. Surgical lung biopsy (SLB) is preferred for diffuse nongranulomatous ILD such as idiopathic pulmonary fibrosis (IPF) or the other idiopathic interstitial pneumonias (IIPs). 
demonstrates a relatively straightforward evaluation for interstitial lung diseases such as IPF.

In general, IPF is a slowly progressive disorder that manifests as progressive dyspnea on exertion and exercise intolerance culminating after a few years in respiratory failure. However, in a minority of patients (perhaps 15\%), the natural history of the disease differs. In such cases, IPF may progress through unexpected episodes of deterioration characterized by acute worsening of exercise intolerance (usually within 30 days before medical evaluation), decreased oxygen hemoglobin saturation and HRCT with new infiltrates and/or ground glass opacification [3]. After exclusion of other causes (e.g., infection, pulmonary emboli and congestive heart failure), this rapid deterioration is likely related to what has been termed as "exacerbation of IPF," an entity that carries a worse prognosis, due to faster progression of disease [20,21]. As was the case in our patient, and because of its importance, patients showing deterioration should be evaluated for exacerbation of IPF. Because IPF is considered a diagnosis of exclusion, tests should be ordered to rule out other conditions such as infection (often by bronchoscopy), thromboembolic disease or congestive heart failure.

Despite the lack of proven effective therapies, there is still much that can be done for patients with IPF. Initially, effort should be given to confirming the diagnosis by reviewing all data available, and by evaluating imaging studies and histology, if available. The need for oxygen supplementation, evaluation for pulmonary rehabilitation and candidacy for lung transplantation (an effective but limited surgical option) should be considered early. Importantly, education about the disease and its prognosis should be provided, and avoidance of toxic agents (e.g., tobacco) should be encouraged. An early 
evaluation for related conditions is also important including pulmonary hypertension [22]. As there are no proven effective medical therapies for IPF, patients should be considered for inclusion in clinical trials. 


\section{MANAGEMENT OF IDIOPATHIC PULMONARY FIBROSIS [10]}

\section{CHALLENGES IN TRANSLATING PRECLINICAL TRIALS TO EFFECTIVE DRUG THERAPIES}

Before the 1990s, many patients with pulmonary fibrosis of unknown etiology were diagnosed with IPF and were often treated with high doses of steroids. However, although some patients showed improvement, others showed continuing deterioration despite similar clinical presentation and treatment. This led many to conclude that patients initially diagnosed with IPF actually suffered from a variety of conditions; some steroid responsive, others less responsive [23]. This new understanding prompted the careful analysis of the histology of subjects diagnosed with IPF, which ultimately led to the classification of the idiopathic interstitial pneumonias, of which IPF is a member [12]. In contrast to other idiopathic interstitial pneumonias, IPF shows a characteristic histological pattern termed usual interstitial pneumonia (UIP). Later clinical studies that considered histological diagnosis showed that patients diagnosed with IPF in association with histology of UIP represented the most common subgroup of patients with idiopathic interstitial pneumonias and, importantly, the one with the worse prognosis. Specifically, although no well-controlled prospective studies were conducted, a general consensus grew about the lack of responsiveness of IPF to steroids. Despite the above, and considering the lack of effective therapies, many patients with IPF are currently treated 
with steroids and/or other immunosuppressants pending the identification of new therapies. It is for this reason that a stronger emphasis is being placed on the conduct of clinical trials designed to evaluate prospectively the safety and effectiveness of new therapies [8]. To date, these efforts have failed to unveil a "magic bullet" but much has been learned through these trials, which have helped shape the standard of care for this condition.

\section{THE SEARCH FOR EFFECTIVE THERAPIES IN IPF}

There are a variety of new studies testing treatment options for IPF. Clinicaltrials.gov, a registry of federally and privately supported clinical trials, lists 90 currently recruiting (active) or recently completed studies of IPF (http://clinicaltrials.gov). The trials listed show how changing ideas about the pathogenesis of this condition have shaped our approach to therapy. For example, it was originally thought that IPF was caused by relentless inflammation, and this explains why early clinical trials often involved anti-inflammatory agents such as corticosteroids. These early studies were often flawed by misclassification of patients with or without IPF, as well as the lack of placebo control groups, but recent publications suggest that there is no apparent benefit of corticosteroid use for treatment of IPF [23]. Subsequent studies testing other anti-inflammatory agents (e.g., etanercept, interferon- $\gamma$ and interferon- $\beta$ ), among others, have also failed to show much benefit $[8,24,25]$ (Tables 1$3)$. 


\section{Table 1}

Drugs found to be ineffective in the treatment of IPF

Medications

IFN- $\gamma^{*}$ vs. placebo $[25]$

Bosentan vs. placebo [26]

\section{Results}

No increase in survival

No change in $6 \mathrm{MWT}^{*}$ seen
Study Comments

New study testing IFN- $\gamma$ by an inhaled route is planned

New study is testing to see whether Bosentan delays time until death/decreases lung deterioration

Imatinib vs. placebo [27] Negative study

Prednisolone + IFN $-\gamma \quad$ Inconclusive results

vs. Prednisolone + Colchicine [28]

NAC*, azathioprine, Increased mortality vs. Trial testing NAC alone vs.

+ prednisone vs. placebo [29] placebo group placebo undergoing

Warfarin vs. placebo [30] Negative study

Warfarin appeared to increase mortality due to respiratory worsening. Didn't include patients requiring warfarin for nonIPF related treatments. 


\section{Table 2}

Drugs requiring further investigation in the treatment of IPF

\begin{tabular}{|c|c|c|}
\hline Medications & Results & Study Comments \\
\hline Etanercept vs. placebo [24] & Improved $\mathrm{FVC}^{*}$ and $\mathrm{DL}_{\mathrm{CO}} *$ & Original study did not reach primary end point \\
\hline $\begin{array}{l}\text { Prednisone }+ \text { azathioprine vs. } \\
\text { prednisone }+ \text { placebo }\end{array}$ & $\begin{array}{l}\text { Trend to improved resting arterial } \\
\mathrm{O}_{2}, \text { small increase in survival }\end{array}$ & $\begin{array}{l}\text { Small test group, only a trend toward } \\
\text { improvement, changes not significant }\end{array}$ \\
\hline $\begin{array}{l}\text { Prednisolone }+ \text { anticoagulant vs. } \\
\text { prednisolone [32] }\end{array}$ & Significant increase in survival & $\begin{array}{l}\text { Not a double-blinded study. Diagnostic criteria } \\
\text { used were not standard }\end{array}$ \\
\hline Pirfenidone vs. placebo [33] & Improved lung function variables & $\begin{array}{l}\text { Primary end points for study were atypical and } \\
\text { were not met. } 22 \% \text { of the patients stopped } \\
\text { participating in the study }\end{array}$ \\
\hline N-acetylcysteine vs. Bromhexine [34] & $\begin{array}{l}\text { No improvement in pulmonary } \\
\text { function tests or quality of life }\end{array}$ & $\begin{array}{l}\text { May delay disease progression in } \mathrm{N} \text {-acetylcysteine } \\
\text { group }\end{array}$ \\
\hline
\end{tabular}




\section{Table 3}

Drugs considered to have some efficacy but require further testing

\begin{tabular}{|c|c|c|}
\hline Medications & Results & Study Comments \\
\hline Pirfenidone vs. placebo [35] & $\begin{array}{l}\text { Less of a decrease in \%FVC*, } \\
\text { Fewer deaths in high dose group }\end{array}$ & $\begin{array}{l}\text { Approved treatment in Japan, more side effects in } \\
\text { high dose group. No FDA* approval }\end{array}$ \\
\hline Prednisolone + IFN $-\gamma^{*}$ vs. prednisolone [36] & $\begin{array}{l}\text { Significant improvement in lung } \\
\text { function for combination drug } \\
\text { patients }\end{array}$ & $\begin{array}{l}\mathrm{N}=18 \text {, significant improvement atypical. } \\
\text { Retesting with a larger patient group advisable } \\
\text { and include a double-blinded, placebo control }\end{array}$ \\
\hline $\begin{array}{l}\text { Azathioprine, prednisone, + NAC* vs. } \\
\text { Azathioprine, prednisolone, + placebo [37] }\end{array}$ & $\begin{array}{l}\text { Decreased decline in FVC and } \\
\text { gas transfer for } \mathrm{CO}^{*} \text { deterioration } \\
\text { in the non-placebo group }\end{array}$ & Lack of a placebo-only control group \\
\hline
\end{tabular}


Knowledge has accumulated suggesting that tissue fibrosis can occur without inflammation or dissociated from it. In fact, in cases where IPF is suspected, significant tissue inflammation should lead clinicians to search for an alternative diagnosis. Despite the above, a statement published by the American Thoracic Society/European Respiratory Society in 2000 suggested the use of low-dose prednisone (a corticosteroid) with azathioprine (an immunosuppressant) for treatment. This combination became a standard therapy largely due to the lack of available treatment options. However, in 2008, the Idiopathic Pulmonary Fibrosis International Group Exploring N-acetylcysteine I Annual Study tested the addition of an antioxidant, $\mathrm{N}$-acetylcysteine, to the standard regimen [37]. Although this study showed delays in the progression of IPF, it was heavily criticized for its small size and for the lack of a control group. Nevertheless, despite the lack of proven effectiveness, this new, largely untested, treatment regimen including $\mathrm{N}$-acetylcysteine (NAC) evolved as the standard of care. Unfortunately, a recent randomized, double-blinded, placebo-controlled trial testing the effect of this combination therapy (prednisone, azathioprine, and NAC) was stopped prematurely due to an increased rate of death in the combination group versus placebo (8 vs. 1 patients). In addition there was no physiological or clinical benefit seen in the combination group and combination therapy patients had increased adverse events [29]. However, a separate study comparing the use of only NAC versus placebo is still ongoing [29]. A similar situation has recently evolved regarding the use of warfarin (an anticoagulant) in the treatment of IPF [38]. Previous findings had suggested a potential benefit of warfarin in a subset of patients (receiving a weak negative recommendation [3]), however, recent evidence has shown that warfarin therapy shows no clinical efficacy and is associated 
with an increased risk of mortality (excluding IPF patients that required anticoagulation for non-IPF related reasons) [30].

As other concepts about IPF pathogenesis emerged, phase I, phase II and phase III clinical trials have been conducted to test their accuracy. In general, the agents tested show benefits when examined in animal models of lung fibrosis. These include inhibitors of fibrotic growth factors (e.g., connective tissue growth factor, transforming growth factor $\beta$ and platelet-derived growth factor), endothelin receptor antagonists (e.g., bosentan), inhibitors of kinases (e.g., imatinib), anticoagulants (warfarin) and antifibrotics (e.g., pirfenidone) [8, 26, 27, 32, 39]. Unfortunately, benefits have not always been observed in these studies, improved survival has not been demonstrated and much controversy still remains. However, the data generated suggest that some of these approaches should be considered further. More importantly, these studies have prompted the generation of new and testable hypotheses about IPF pathogenesis.

There are several important factors to consider when designing a new study. Of particular importance is the use of a placebo-only control group. Also, a standard or at least a minimum criterion for primary endpoints should be established. Although enhanced survival is the most robust endpoint for a trial, other factors such as a change in forced vital capacity or performance on 6-minute walk test and possibly the use of biomarkers (once suitable markers are identified) should be considered for use as primary and secondary endpoints.

Clinical studies are often limited by a small sample size, especially in the case of a disease such as IPF. In part, the limited number of patients available for participation in 
trials is addressed by involving multicenter clinical trials. Collaboration between different centers can and should be encouraged. Recruitment criteria are established and include confirmation of a diagnosis of IPF in all the participating patients. Until effective treatments are found, attention should be given to the treatment of comorbid conditions. A recent clinical trial tested the ability of Sildenafil, a phosphodiesterase- 5 inhibitor, to improve patients with advanced IPF. The primary outcome was not achieved; however, small but significant differences in arterial oxygenation, carbon monoxide diffusion capacity, degree of dyspnea and measures of quality of life were seen [13].

IPF, as the name suggests, has no known cause. Nevertheless there are several factors that are associated with a greater risk for disease development and progression including male gender, genetic mutations, smoking, and gastrointestinal reflux disease [3, 40-42]. In certain subjects, pulmonary fibrosis develops from known pro-fibrotic stimuli such as allergens, asbestos, and drugs, or is associated with chronic inflammatory conditions such as connective tissue disorders [43, 44].

\section{THE IMPACT OF GENETICS}

A genetic association of IPF is seen in less than $5 \%$ of all patients with the disease although higher numbers $(\sim 20 \%)$ have been reported in some smaller cases. The familial form, as defined by affecting two or more members of the same biological family, is histologically and clinically identical to the sporadic form of IPF [45]. Evidence now suggests that mutations in two separate gene pathways both lead to the common phenotype of IPF. These mutations occur in the genes encoding lung surfactant proteins 
$\mathrm{C}$ and $\mathrm{A} 2$ which lead to increased endoplasmic reticulum stress in type 2 alveolar epithelial cells (these mutations are found almost exclusively in familial cases of IPF). The other mutations occur in genes that encode telomerase (TERT and TERC) which are believed to cause IPF by the shortening of telomere lengths in lung stem cells (the majority of these cases are found in familial IPF). Interestingly, short telomere lengths are found in both familial and sporadic IPF even in the absence of the telomerase mutation. This suggests a connection with the age-related prevalence of IPF [46]. It is important to note that the surfactant and telomerase mutations account only for $\sim 15-20 \%$ of all familial IPF cases. Thus, there are likely several other genes responsible for the development of IPF. In addition, although these mutations are significantly less prevalent in sporadic IPF, the pathways that are involved with these genes are often dysfunctional [47].

Race appears to play a role in patient survival and outcome. The three-year survival rate was found to be $\sim 45 \%$ for Non-Hispanic whites versus $\sim 35 \%$ for NonHispanic Blacks and Hispanics [48]. In addition, race has an effect on survival following lung transplantation. Black and Hispanic patients with advanced IPF have shorter survival times following lung transplantation. The 2-year survival estimate for Blacks and Hispanics was $30 \%$ versus $82 \%$ in Whites and Asians [49].

\section{OTHER IMPORTANT CONSIDERATIONS REGARDING IPF MANAGEMENT}

The lack of Federal Drug Administration-approved medications for the treatment of IPF does not mean that physicians have nothing to offer in this setting. This is far 
from the case considering that lung transplantation and oxygen supplementation have been shown to extend survival in these patients [50]. In addition, these and other interventions can improve quality of life. Thus, it is important that patients suspected and/or diagnosed with IPF be referred to centers with expertise in this field, so that patients are evaluated thoroughly and appropriate treatment strategies are considered. The general management of IPF is summarized in Figure 3 and specific interventions are described later.

\section{OXYGEN THERAPY}

As the disease progresses, IPF causes dyspnea on exertion mainly due to increases in the work of breathing and alterations in gas exchange capacity and hypoxemia. In this setting, supplemental oxygen therapy is useful as it may diminish dyspnea and improve exercise tolerance. However, the need for supplemental oxygen may be missed if patients have only resting arterial blood gas or oxygen saturation measurements. Patients should be tested for supplemental oxygen using a 6-minute walk test, a test that evaluates distance walked and hemoglobin oxygen saturation during exertion. Oxygen supplementation should be considered in patients who show hemoglobin oxygen desaturation below $88 \%$ (or $\mathrm{Po}_{2}<55 \mathrm{~mm} \mathrm{Hg}$ ) [3]. Oxygen can be given nasally, through a mask or in some cases by transtracheal delivery. The amount of time required to use the oxygen delivery system depends on the condition of the patient's disease. Initially, it may only be required during strenuous activities, but as the disease progresses, it may be required more often or continuously. Oxygen may be supplied by a stationary

concentrator for home use and portable tanks of compressed gas. When higher flow rates 


\section{Figure 3}

General management of patients with IPF

\section{Management of IPF}

$\begin{array}{cc}\begin{array}{c}\text { Confirm diagnosis } \\ \text { Educate about } \\ \text { prognosis }\end{array} & \begin{array}{c}\text { Consider if candidate } \\ \text { for pulmonary rehabilitation }\end{array} \\ \text { Stop offenders } & \begin{array}{c}\text { Determine candidacy } \\ \text { for lung transplantation }\end{array} \\ \begin{array}{c}\text { Evaluate for } \\ \text { oxygen } \\ \text { supplementation }\end{array} & \begin{array}{c}\text { Evaluate other problems } \\ \text { (e.g., cough, pulmonary } \\ \text { hypertension) }\end{array} \\ & \text { Consider clinical trial }\end{array}$

Once identified, patients suspected of IPF should be evaluated to confirm the diagnosis. If confirmed, attention should be given to education and evaluation for the need for oxygen supplementation and candidacy for pulmonary rehabilitation and lung transplantation. Control of cough and early detection of associated conditions (eg, pulmonary hypertension) should be considered. Finally, until an effective drug becomes available, patients should consider participating in clinical trials. 
(4-5 L/min) are needed at rest or long portable times are required, a liquid oxygen setup is appropriate.

\section{PULMONARY REHABILITATION}

The benefits of physical rehabilitation have not been widely studied; however, pulmonary rehabilitation is considered important in building and maintaining aerobic and physical fitness and treatment of dyspnea in select patients [51]. Training usually involves breathing exercises, physical conditioning and anxiety and depression management. Similar to the necessity of talking to patients who convey an understanding of their treatment options, pulmonary rehabilitation is essential in helping patients manage their condition and maintain the highest quality of life possible.

\section{COUGH SUPPRESSION}

One of the most distressing and difficult-to-treat consequences of IPF is cough. Cough has a measureable impact on the quality of life and correlates with cough frequency as determined by cough counts or perception of cough frequency by patients [52]. The mechanisms of cough in IPF are not well understood, but the triggers for this symptom reside in the airways as demonstrated by cough challenge tests using capsaicin. Patients with IPF show a 30 -fold increased sensitivity (i.e., 30 times lower dose versus healthy subjects) to cough inducing levels of capsaicin [53]. Even though chronic cough is a major symptom of IPF, approximately one half of the cases of chronic cough in IPF can be attributed to more common causes such as hyperresponsive airways, gastroesophageal reflux and rhinosinusitis [54]. The management of cough in IPF should, therefore, include a careful evaluation for the other causes of chronic cough. 
Treatment of chronic cough due to IPF is difficult, and common treatments often fail. Ironically, systemic steroids that have no impact on the course of IPF may be effective in mitigating cough in this disease [55].

\section{LUNG TRANSPLANTATION}

Lung transplantation is the closest option to a cure for IPF; however, there are several limitations to this treatment option. IPF is a disease that is predominantly associated with older age with the median age being 66 years at diagnosis [56]. Advanced age, along with the frequency of comorbid conditions (i.e., pulmonary arterial hypertension, cardiac disease) increase the complications and risks associated with surgery [57]. This also results in worse outcomes for IPF patients following transplantation compared with other respiratory conditions [58].

\section{SUMMARY}

IPF is a chronic disease characterized by progressive lung fibrosis. Studies in animals have unveiled drugs that might be helpful in preventing lung fibrosis. Unfortunately, these studies have not been duplicated in humans, and, to date, clinical trials have failed to unveil a drug capable of improving survival. Consequently, lung transplantation and oxygen supplementation remain the only therapeutic options capable of improving survival in patients with IPF. Despite this, there is still much that can be done to improve the quality of life in these patients including physical rehabilitation and control of cough (Figure 3). Also, patients may benefit from the early detection and 
treatment of secondary problems (e.g., pulmonary hypertension). Until a therapy is found, patients with IPF are encouraged to engage in well-conducted clinical trials that may someday unveil a safe and effective treatment for this devastating disease. 


\section{IMPACT TO HEALTHCARE National Database (Healthcare Cost and Utilization Project)}

The burden placed on the healthcare system by patients with IPF is extensive. IPF patients are some of the most costly patients to treat during advanced stages of the disease. Limited treatment options and the lack of FDA approved drugs make understanding the impact of IPF patients to the healthcare system essential. In order to bridge the knowledge gap between the clinical arena and the costs associated with treating patients at the national level an epidemiological study using a national healthcare database was undertaken focusing on hospitalization rates and costs.

\section{HOSPITALIZATION RATES, LENGTH OF STAY, MORTALITY AND COSTS OF IPF IN THE UNITED STATES}

The incidence of IPF appears to be increasing although questions remain as to whether this is related to increased awareness or a true change in incidence of the disease. This has been influenced by the increased use of high-resolution computed tomography scans, which became more commonplace since the 1990s and provided an easier, more economical, and more accurate means of diagnosing IPF and related pulmonary disorders [59]. Additionally, the publication by the American Thoracic Society and European Respiratory Society (ATS/ERS) of an international consensus statement of the diagnosis and treatment guidelines for IPF in 2000 [60] and of idiopathic interstitial pneumonias in 2002 [12] likely increased physician awareness and accurate diagnosis of the disease. 
Current information about the morbidity and mortality of pulmonary fibrosis is needed. Others have reported a seasonal variation in mortality due to IPF (mortality is the highest during the winter) [61] and have reported the rate of mortality increasing between 1992 and 2000; the average age- and sex-adjusted mortality rate was 50.8 per 1,000,000 people [62]. Furthermore, among those with IPF, Blacks are less likely to die from IPF than Whites, whereas Hispanics are more likely to die than Whites [63].

Our analysis focuses on hospital admissions, in-hospital mortality, length of stay, and charges associated with pulmonary fibrosis. For this, we utilized a public database containing hospitalization information known as the Healthcare Cost and Utilization Project (HCUP) Public Database.

\section{METHODS}

\section{THE HCUP DATABASE}

Data available through HCUP provide access to a family of healthcare databases. These include the Nationwide Inpatient Sample (NIS), which contains inpatient data from a national sample of over 1,000 hospitals in the United States as well as the State Inpatient Database (SID) that contains inpatient discharge abstracts from participating states. Data from the databases are provided from 1988 to the present date [11]. The NIS is the largest all-payer inpatient care database in the United States and contains data from $\sim 8$ million hospital stays yearly. The NIS contains data from 1,050 hospitals located in 44 states and approximates a $20 \%$ stratified sample of U.S. community hospitals. The SID contains the inpatient discharge abstracts from participating states. The SID encompasses $\sim 90 \%$ of all U.S. community hospital discharges. 


\section{PATIENT COHORT AND DATA ANALYSIS}

Using the HCUPnet Database, we analyzed data related to ICD-9-CM codes for post inflammatory pulmonary fibrosis (PPF, ICD-9 code 515), idiopathic fibrosing alveolitis (IFA, ICD-9 code 516.3), and sarcoidosis (ICD-9 code 135). We compared different parameters available for each disease (e.g., charges, length of stay, mortality, procedures, and diagnoses). We investigated changes over time for the United States (for those states participating in the database) between 1993 and 2008. Geographic location was defined as Northeast, South, Midwest, and West based on U.S. census regions. Classification of co-morbidities was based on the patient's secondary diagnoses.

\section{RESULTS}

\section{HOSPITAL ADMISSIONS}

Analysis of the HCUPnet Database revealed an increase in the number of hospital discharges nationwide between 1993 and 2008 with an upsurge by the turn of the century (Figure 4A). During the same time period, a steady increase in hospital admissions was noted for patients with a principal diagnosis of PPF, doubling in the same time period and reached close to 30,000 admissions per year in 2008 ; these changes averaged to a $4.9 \%$ increase per year (Figure 4B). In contrast, admissions related to IFA were relatively stable, declined early in the past decade to 3,513 admissions per year in 2008 . For comparison, hospital admissions related to sarcoidosis have increased on average 
Figure 4

Hospital Discharges

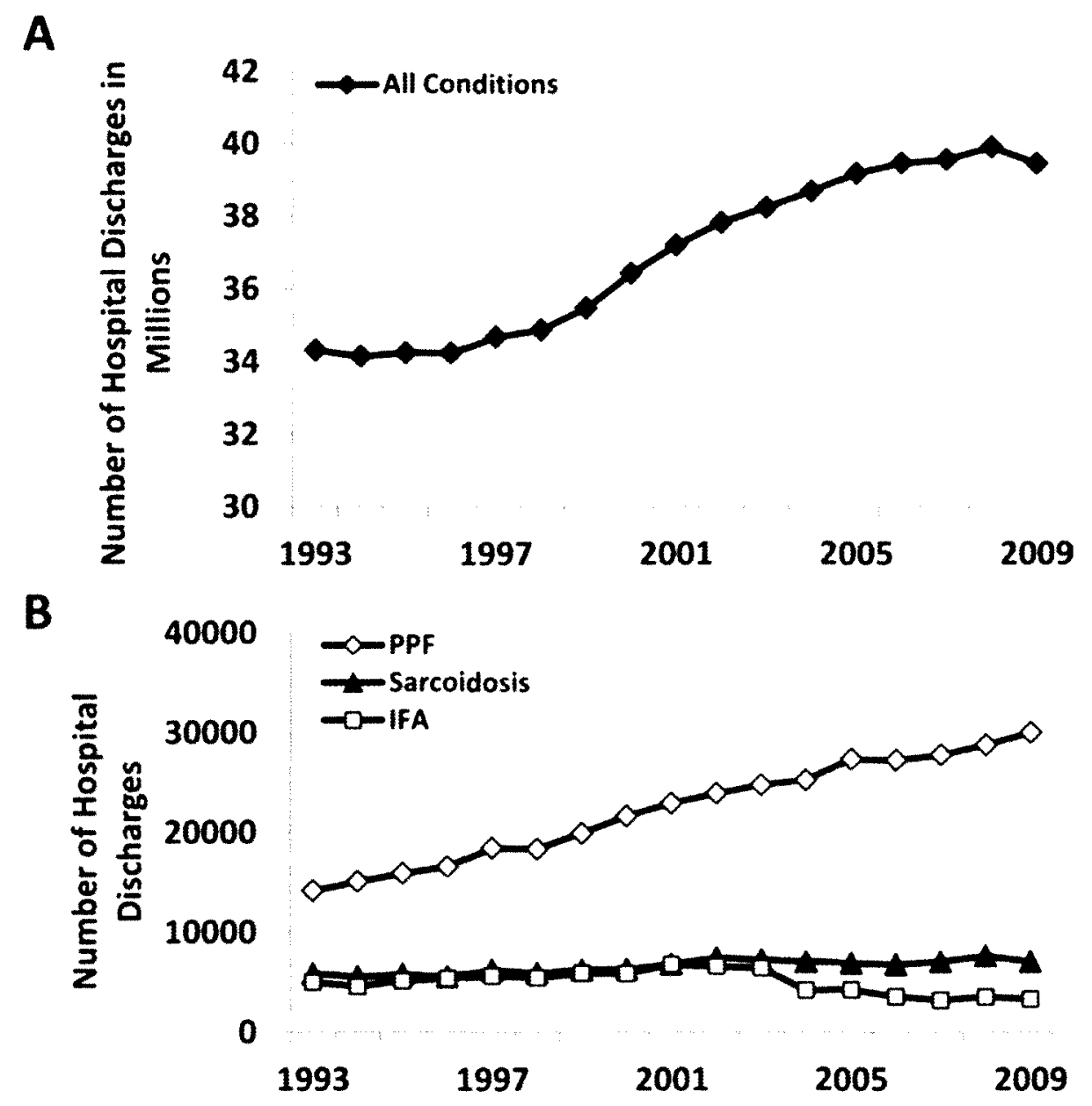

Figure $4 \mid \mathbf{A}$. Increase in the number of hospital discharges nationwide. B. Hospital discharges have doubled for PPF. Discharges for IFA have decreased, while sarcoidosis discharges have increased $1.9 \%$ /year on average.

IFA, idiopathic fibrosing alveolitis; PPF, postinflammatory pulmonary fibrosis 
1.9\%/year, reaching 7,588 admissions in 2008. Table 5 lists the top procedures performed in these patients with lobectomy or pneumonectomy, diagnostic bronchoscopy/biopsy of bronchus and related procedures, and intubation/mechanical ventilation at the top of the list. Importantly, a significant percentage of patients admitted with a primary diagnosis of PPF, IFA or sarcoidosis also suffered from other important medical conditions, such as essential hypertension, hyperlipidemia, diabetes, and coronary atherosclerosis (Table 6). The database also included information related to adverse effects of medical drugs (E code), showed that up to $16 \%$ of patients hospitalized for IFA suffered from this problem, which was associated with $18.63 \%$ mortality; no information about adverse effects of medical drugs was provided for PPF or sarcoidosis.

When the HCUPnet Database was analyzed to evaluate the reasons for hospitalization, we noted that, in general, patients were hospitalized with primary diagnoses other than PPF, IFA or sarcoidosis. For example, there were many more patients hospitalized with a secondary diagnosis of IFA than those hospitalized with IFA as a primary diagnosis (Figure 5A). Discharges of IFA patients where IFA was not the principal diagnosis decreased significantly between 2003 and 2004 and reached 15,105 by 2008 . Overall, during the 15 year period examined, there was an increase in hospitalizations for patients with PPF, where PPF was not the principal diagnosis; from 150,000 in 1993 to 250,000 in 2008 . As observed for PPF, hospitalizations of patients with secondary diagnosis of sarcoidosis increased steadily in the same time period tripling since 1993 (28,638 hospitalizations) to 74,630 admissions by 2008 (Figure 5C). 
Table 5

Primary procedures for patients hospitalized with IFA*, PPF*, and sarcoidosis $(2009)^{* *}$

\begin{tabular}{|c|c|c|c|c|}
\hline \multirow{2}{*}{$\begin{array}{l}\text { CCS } \\
\text { Code }\end{array}$} & \multirow[t]{2}{*}{ Primary Procedure } & \multicolumn{3}{|c|}{ Principal Diagnosis: \% Total Discharges (\% Mortality) } \\
\hline & & IFA & PPF & Sarcoidosis \\
\hline 36 & $\begin{array}{l}\text { Lobectomy or } \\
\text { Pneumonectomy }\end{array}$ & 9.0 & $20.7(1.9)$ & 5.1 \\
\hline 37 & $\begin{array}{l}\text { Diagnostic Bronchoscopy } \\
\text { and Biopsy of Bronchus }\end{array}$ & 5.4 & 6.8 & 11.5 \\
\hline 38 & $\begin{array}{l}\text { Other Diagnostic Procedures } \\
\text { on Lung and Bronchus }\end{array}$ & 5.6 & $8.8(3.1)$ & 2.0 \\
\hline 216 & $\begin{array}{l}\text { Respiratory Intubation and } \\
\text { Mechanical Ventilation }\end{array}$ & $5.8(67.1)$ & $4.1(49.2)$ & 3.1 \\
\hline 40 & $\begin{array}{l}\text { Other Diag. Procedures of } \\
\text { Respiratory Tract }+ \text { Mediastinum }\end{array}$ & -- & 0.5 & 8.8 \\
\hline
\end{tabular}

Note: $A \geq \mathbf{5} \%$ discharges was used as the threshold for inclusion.

* IFA, idiopathic fibrosing alveolitis; PPF, postinflammatory pulmonary fibrosis

$* *[11]$ 


\section{Table 6}

Secondary diagnoses for patients hospitalized with idiopathic fibrosing alveolitis (IFA)*, postinflammatory pulmonary fibrosis(PPF)* or sarcoidosis (2009) [11]

\begin{tabular}{|c|c|c|c|c|}
\hline \multirow{2}{*}{$\begin{array}{l}\text { CCS } \\
\text { Code }\end{array}$} & \multirow[t]{2}{*}{ Secondary Diagnosis } & \multicolumn{3}{|c|}{ Principal Diagnosis: \% of Total Discharges (\% Mortality) } \\
\hline & & IFA $^{*}$ & $\mathbf{P P F}^{*}$ & Sarcoidosis \\
\hline 131 & $\begin{array}{l}\text { Respiratory Failure, } \\
\text { Insufficiency, Arrest (adult) }\end{array}$ & $47.8(25.2)$ & $30.1(18.2)$ & $14.5(6.3)$ \\
\hline 98 & Essential Hypertension & $44.0(14.1)$ & $42.6(5.8)$ & 39.7 \\
\hline 53 & Hyperlipidemia & $36.3(15.6)$ & $28.2(5.1)$ & 18.3 \\
\hline \multirow[t]{2}{*}{127} & Chronic Obstructive & $35.3(9.7)$ & $34.4(6.2)$ & 18.4 \\
\hline & Pulmonary Disease + Bronchie & asis & & \\
\hline 49 & $\begin{array}{l}\text { Diabetes Mellitus } \\
\text { Without Complication }\end{array}$ & $31.5(12.1)$ & $24.1(7.6)$ & 23.2 \\
\hline 101 & Coronary Atherosclerosis & $31(16.1)$ & $24.5(7.6)$ & -- \\
\hline 257 & Other Aftercare & $29.5(17.5)$ & $20.3(13.7)$ & -- \\
\hline 106 & Cardiac Dysrhythmias & $28.8(23.2)$ & $21.5(11.2)$ & 13.3 \\
\hline 663 & $\begin{array}{l}\text { Screening/History of Mental } \\
\text { Health and Substance Abuse }\end{array}$ & $28.5(14.7)$ & $29.9(4.9)$ & -- \\
\hline 259 & Residual Codes, Unclassified & $27.8(15.1)$ & $25.5(6.4)$ & 27.2 \\
\hline 103 & Pulmonary Heart Disease & $27.7(15.8)$ & $17.4(12.8)$ & 13.4 \\
\hline 138 & Esophageal Disorders & $26.7(12.7)$ & $23(5.3)$ & \\
\hline 108 & $\begin{array}{l}\text { Congestive Heart Failure, } \\
\text { Non-hypertensive }\end{array}$ & $26.4(12.7)$ & $23.1(11.4)$ & 13.1 \\
\hline 55 & Fluid and electrolyte disorders & $26(25)$ & $20.0(13.5)$ & 17.7 \\
\hline 122 & Pneumonia (not TB/STDs) & $25.2(22.6)$ & $18.7(16.4)$ & 14.6 \\
\hline 244 & $\begin{array}{l}\text { Other Injuries/Conditions } \\
\text { Due to External Causes }\end{array}$ & $24.9(17.5)$ & $18.3(11.5)$ & -- \\
\hline 133 & $\begin{array}{l}\text { Other Lower Respiratory } \\
\text { Disease }\end{array}$ & $23.1(13.6)$ & $16.5(7.5)$ & 65 \\
\hline 59 & Anemia & $21.7(21.2)$ & $18.2(9.4)$ & 17.6 \\
\hline 2617 & $\begin{array}{l}\text { E Codes: Adverse Effects } \\
\text { of Medical Drugs }\end{array}$ & $16.1(18.6)$ & -- & -- \\
\hline
\end{tabular}




\section{Figure 5}

Hospital Discharges by Disease Condition

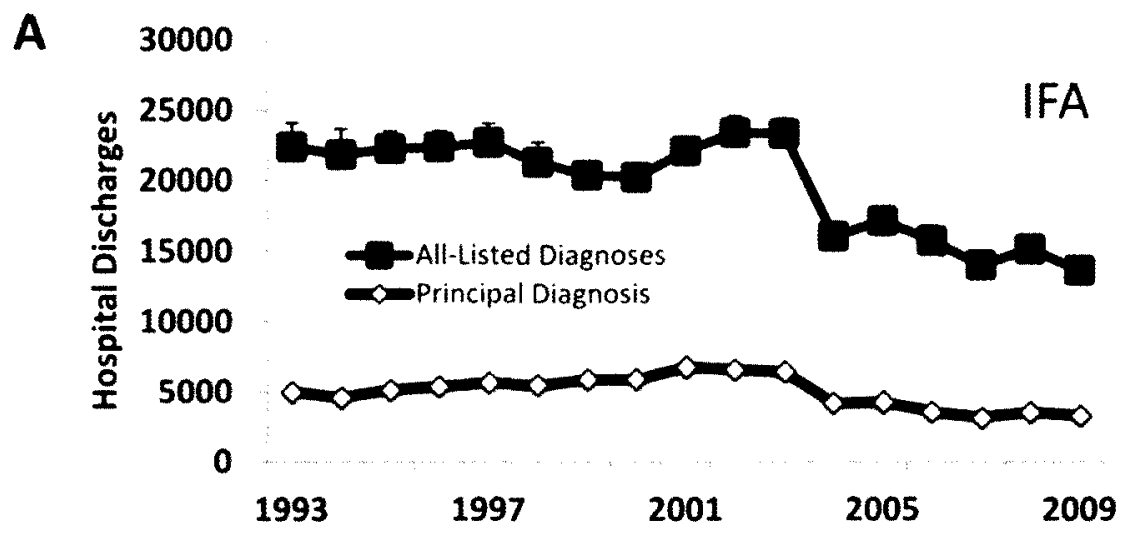

B
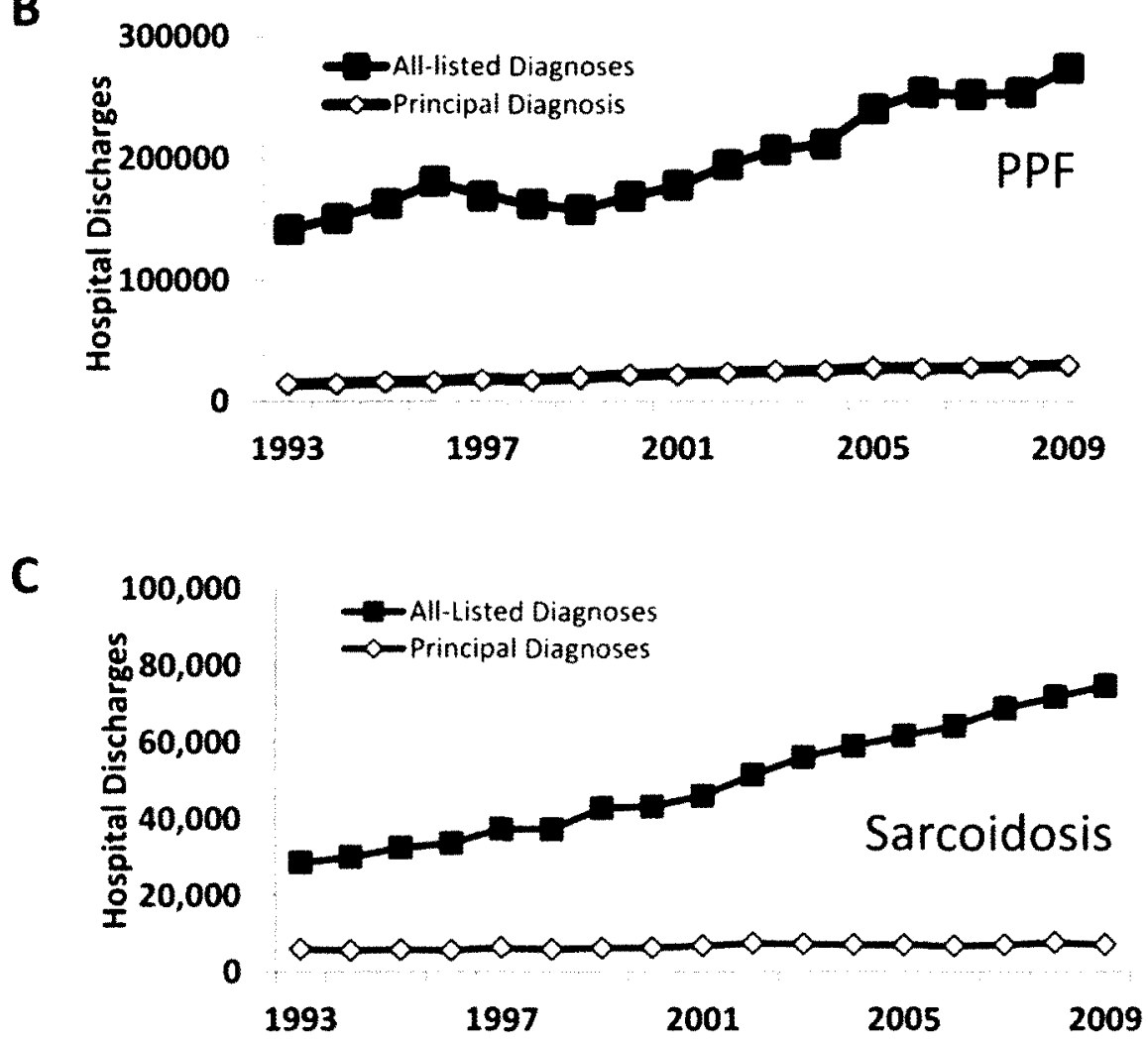

Figure $5 \mid$ A. Discharges of IFA patients where IFA was not the principal diagnosis decreased significantly between 2003 and 2004. B. Discharges where PPF was not the principal diagnosis increased overall from 150,000 to 250,000 . C. Discharges for sarcoidosis have increased from 3,513 to 7,588 .

IFA, idiopathic fibrosing alveolitis; PPF, postinflammatory pulmonary fibrosis 
The above data suggested that patients with diagnosis of PPF, IFA or sarcoidosis are often admitted due to other conditions. Table 7 lists the top primary diagnoses for which patients were admitted, pneumonia, respiratory failure, chronic obstructive pulmonary disease, congestive heart failure and septicemia led the list. When data were available for an individual diagnosis (i.e., pneumonia), it was evident that subjects with IFA experienced the highest mortality from these conditions followed by PPF. Also, subjects with IFA and PPF showed the highest incidence for critical secondary diagnoses, such as respiratory failure.

\section{LENGTH OF STAY}

Length of stay during hospitalization for all conditions decreased steadily in the mid1990s and stabilized at 4.6 days by 2009 (Figure 6A). Length of stay for PPF, IFA and sarcoidosis were higher (1-3 days) than the average for all conditions during all years of this analysis. For PPF, length of stay decreased during the mid-1990s and remained relatively stable at 6 days. On the other hand, length of stay for IFA was the highest of this group starting at 9.7 in 1993, decreasing thereafter, only to increase again in 2007 with an average length of stay of 9.3 days by 2008 . For sarcoidosis, length of stay declined progressively from 7.5 days in 1993 to 6.2 days by 2008 . As shown in Figure $6 \mathrm{~B}-\mathrm{D}$, length of stay for the three diagnoses did not appear to differ significantly based on region except for IFA for which the length of stay increased dramatically in 2007 with a return to baseline a year later in the Northwest region. 


\section{Table 7}

Primary diagnoses for patients hospitalized with secondary diagnoses of IFA*, PPF*, or sarcoidosis $(2009)^{* *}$

\begin{tabular}{|c|c|c|c|c|}
\hline \multirow{2}{*}{$\begin{array}{l}\text { CCS } \\
\text { Code }\end{array}$} & \multirow[t]{2}{*}{ Primary Diagnosis } & \multicolumn{3}{|c|}{ Secondary Diagnosis: \% of Total Discharges (\%Mortality) } \\
\hline & & IFA & PPF & Sarcoidosis \\
\hline 122 & Pneumonia & $14.6(10.4)$ & $12.8(7.1)$ & $6.5(1.9)$ \\
\hline 131 & $\begin{array}{l}\text { Respiratory Failure, } \\
\text { Insufficiency, Arrest (adult) }\end{array}$ & $10.5(31.7)$ & $6.2(21.7)$ & $2.6(11.2)$ \\
\hline 127 & $\begin{array}{l}\text { Chronic Obstructive Pulmonary } \\
\text { Disease and Bronchiectasis }\end{array}$ & 8.1 & $9.8(2.2)$ & 3.7 \\
\hline 108 & $\begin{array}{l}\text { Congestive Heart Failure, } \\
\text { Non-hypertensive }\end{array}$ & 7.0 & $8.2(5.4)$ & $4.4(3.2)$ \\
\hline 2 & Septicemia (except in labor) & $5.0(25.2)$ & $4.2(22.7)$ & $3.1(10.3)$ \\
\hline 106 & Cardiac Dysrhythmias & 2.9 & $3.1(3.3)$ & 2.8 \\
\hline 100 & Acute Myocardial Infarction & 2.2 & $2.2(9.3)$ & -- \\
\hline 128 & Asthma & 1.5 & 2.0 & 3.7 \\
\hline
\end{tabular}

*IFA, Idiopathic Fibrosing Alveolitis; PPF, Postinflammatory Pulmonary Fibrosis **[11] 
Figure 6 Length of Stay

A

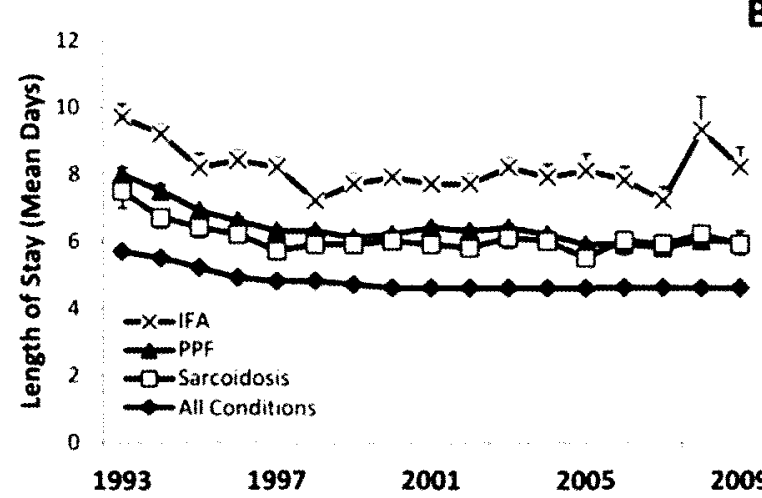

16

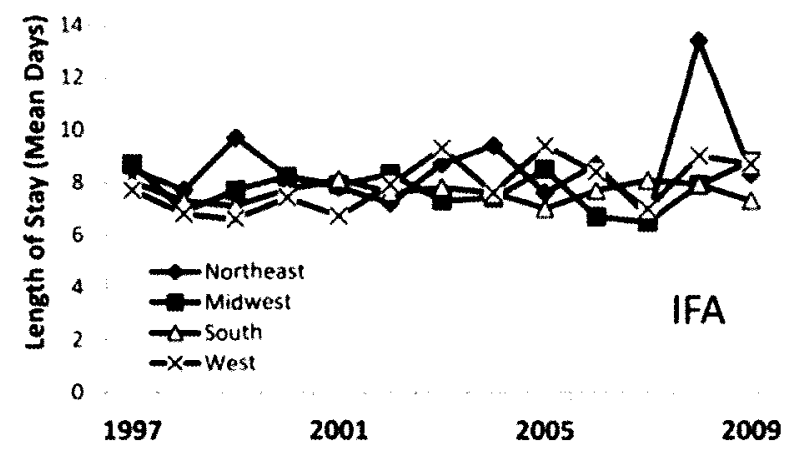

\section{C}
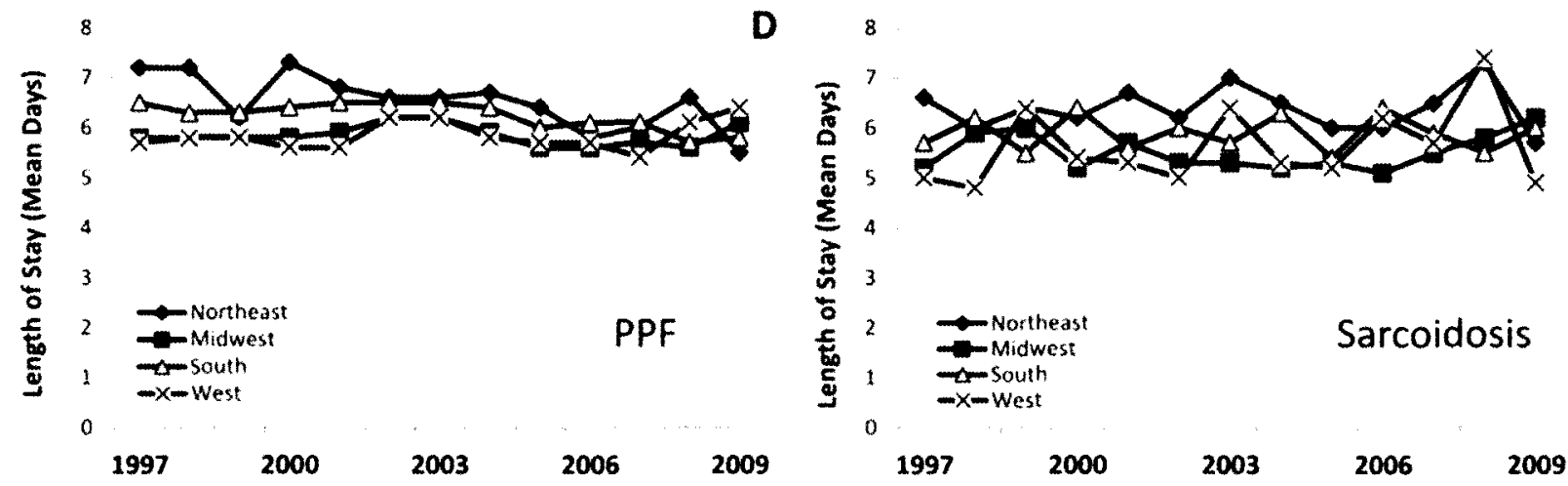

Figure $6 \mid$ A. Length of stay during hospitalization for all conditions decreased steadily in the mid-1990s and stabilized at 4.6 days by 2009. PPF and sarcoidosis had similar average lengths of stay, whereas IFA patients had the longest ( $\sim 9$ days). B-C. Length of stay for the three diagnoses did not appear to differ significantly based on region except for IFA for which the length of stay increased dramatically in 2007 with a return to baseline a year later in the Northwest region.

IFA, idiopathic fibrosing alveolitis; PPF, postinflammatory pulmonary fibrosis; Region is defined by the U.S. Census regions 
The length of stay was also analyzed based on the payer (Figure 7A-C). For the duration of the time studied, patients paying with private insurance had the shortest length of stay for patients with PPF and sarcoidosis ( $\sim 5$ days for each condition). The length of stay varied for IFA, although Medicaid patients generally stayed the longest time in the hospital ( $\sim 10$ days). Medicaid patients with PPF also had the longest length of stay as compared to other payer types ( $\sim 7$ days). Length of stay did not appear to differ significantly when the data were analyzed for gender (Figure 8A-C). However gender did play a role on percent hospital discharges. We saw more female discharges from 1997-2002 for IFA, but these values switched in recent years with males comprising the majority of hospital discharges (Figure 9A). For PPF and sarcoidosis, there was a $\sim 10$ to $35 \%$, respectively, greater percent of female discharges compared to males (Figure 9BC). IFA discharges by gender were not as distinct.

We then determined where the patients were sent after their hospitalization. As depicted in Figure 10, most patients with PPF ( $65 \%)$, IFA (50\%), and sarcoidosis $(\sim 80 \%)$ demonstrated a 'routine discharge' indicating that they returned home with routine care. However, this number decreased with time, and by 2009 , over $15 \%$ of patients hospitalized for PPF and IFA were discharged to home health care (Figure 10B). A smaller percentage was discharged to another short-term hospital (Figure 10C). For PPF and IFA, between $10 \%$ and $15 \%$ of patients were discharged to another institution, such as a nursing home or rehabilitation center; this category has increased $1.8 \%$ for both conditions over the time period examined (Figure 10D). 


\section{Figure 7}

Length of Stay by Payer

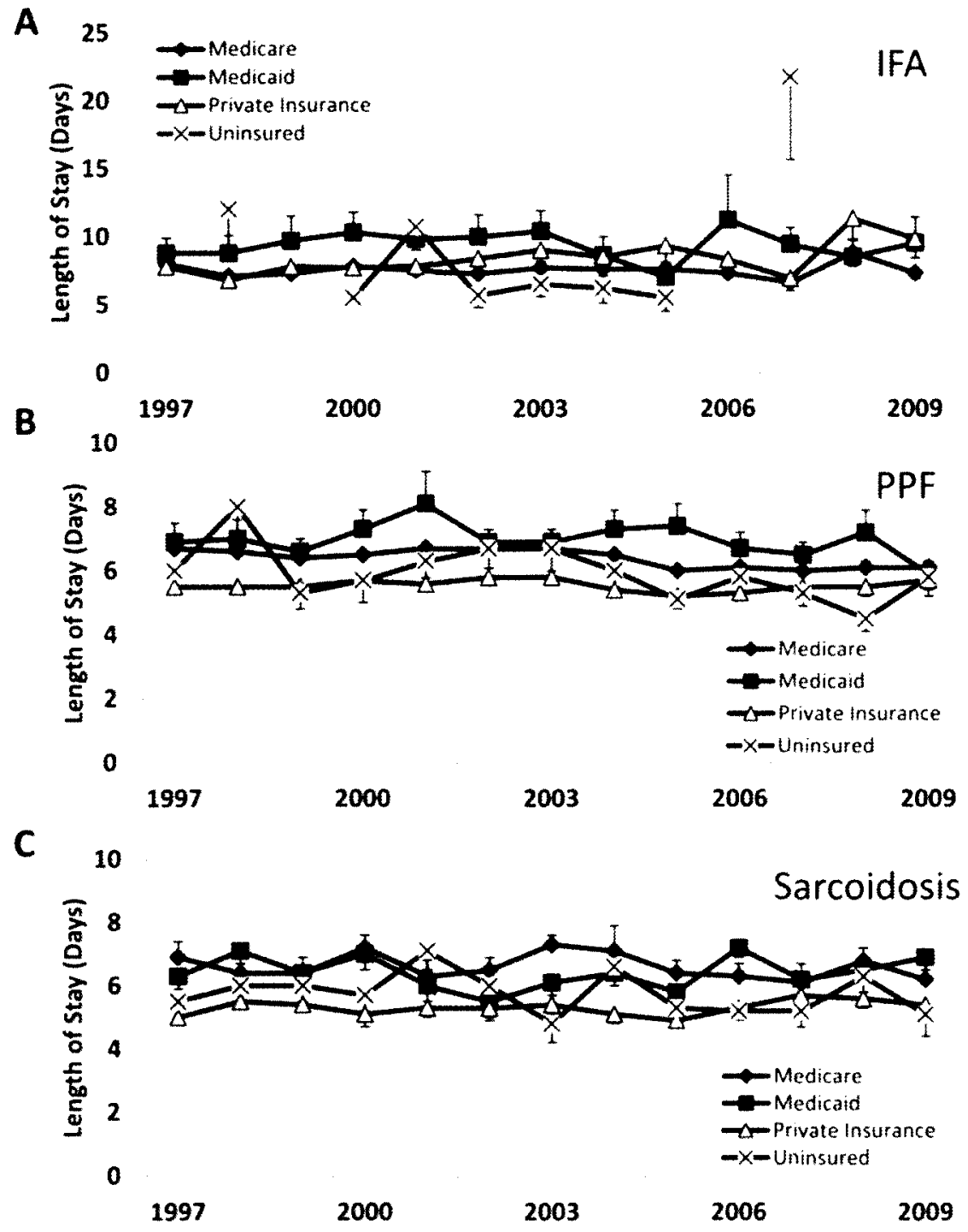

Figure $7 \mid$ A. LOS varied for IFA, although Medicaid patients generally stayed the longest time in hospital ( 10days). B. Medicaid patients with PPF also had the longest LOS on average ( 7 days). B-C. Private insurance had the shortest length of stay on average for PPF and sarcoidosis patients ( $\sim 5$ days for each condition).

IFA, idiopathic fibrosing alveolitis; PPF, postinflammatory pulmonary fibrosis; LOS, length of stay 
Figure 8

Length of Stay by Gender

A

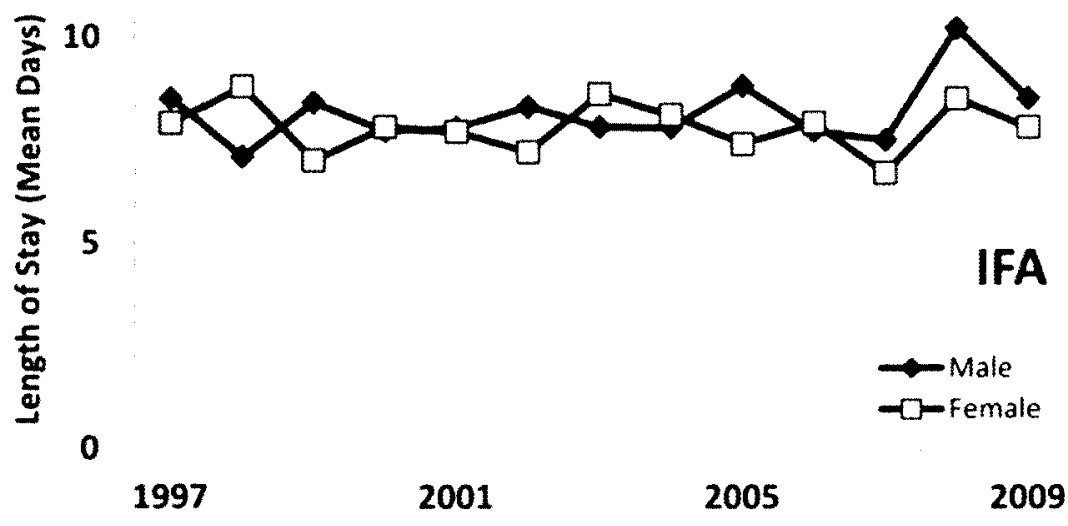

B
8

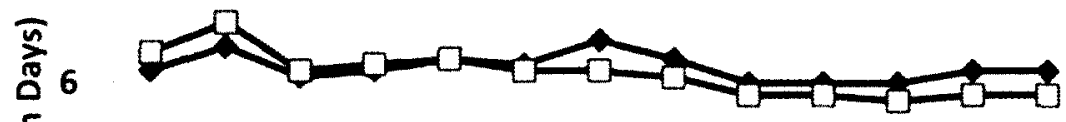

密 4

C

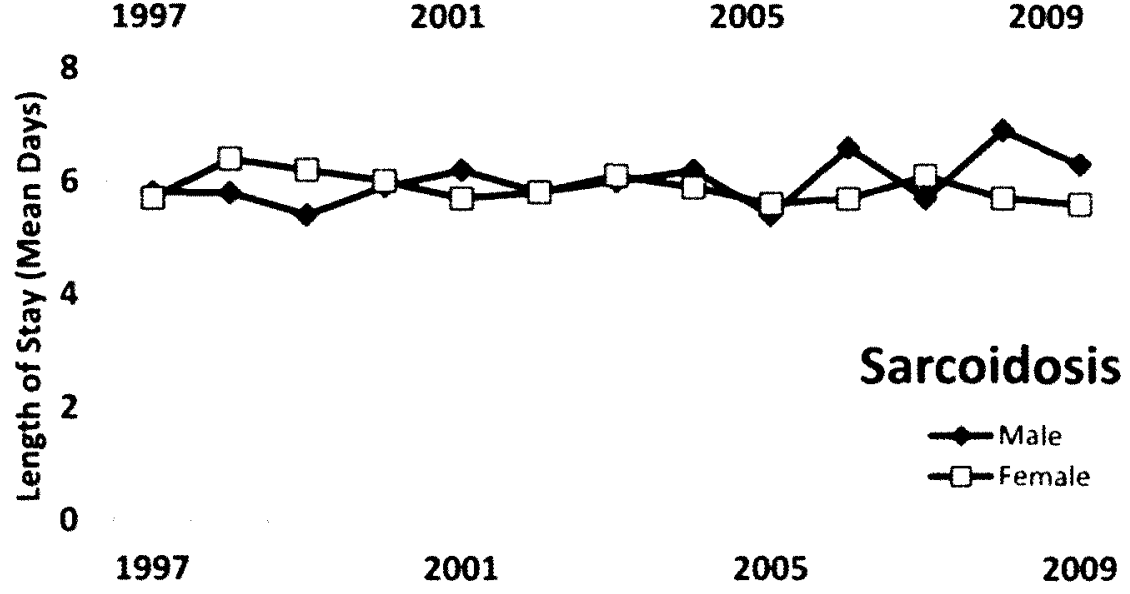

Figure $8 \mid$ A-C. Length of stay did not vary greatly when analyzed by gender for each disease.

IFA, idiopathic fibrosing alveolitis; PPF, postinflammatory pulmonary fibrosis 


\section{Figure 9}

Discharges by Gender
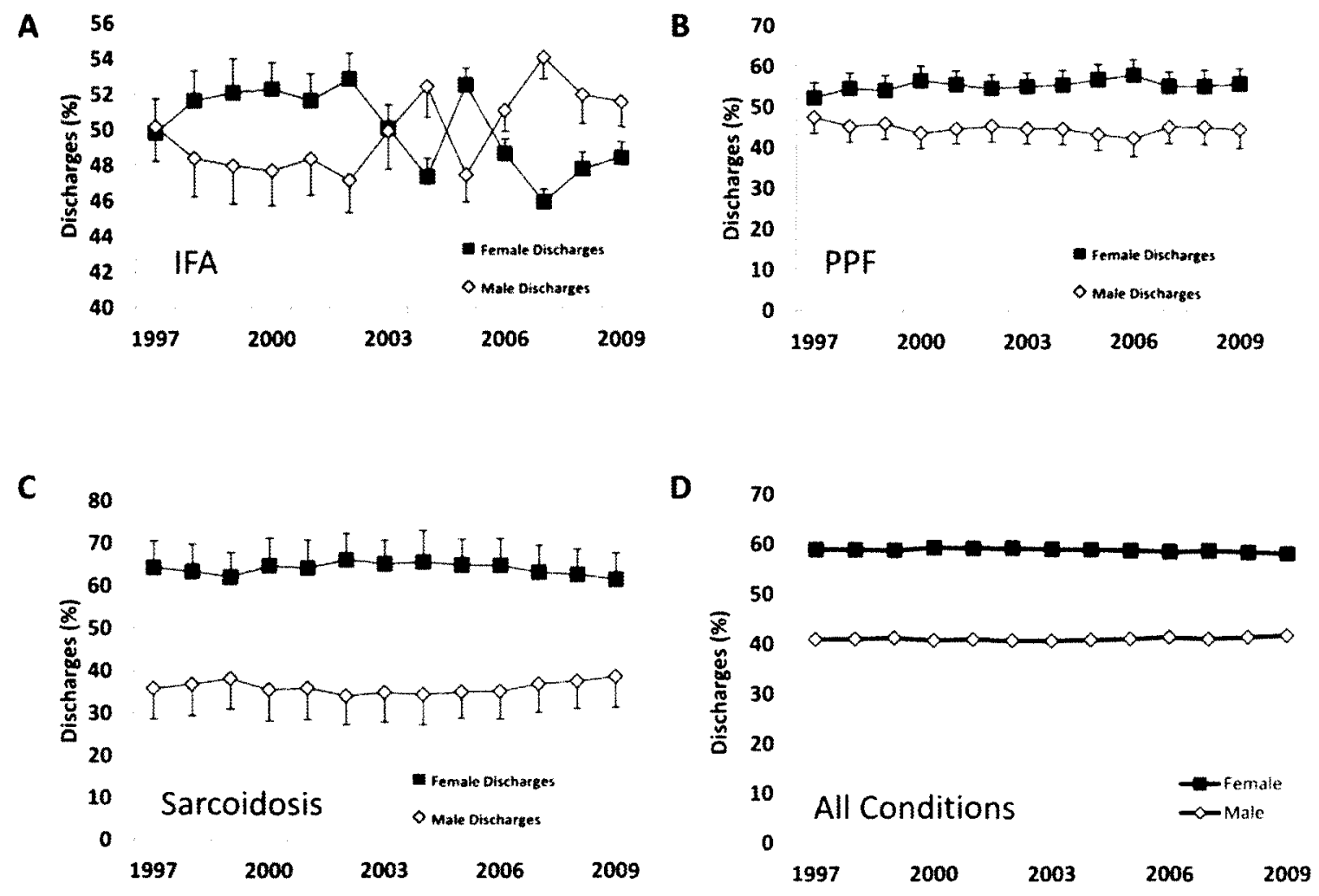

Figure $9 \mid$ A. There were more female discharges from 1997-2002 for IFA, but these values switched in recent years with males comprising the majority of hospital discharges. B-C. For PPF and sarcoidosis, there was a $\sim 10$ to $35 \%$, respectively, greater percent of female discharges compared to males. Comparatively, IFA discharges by gender were not as distinct.

IFA, idiopathic fibrosing alveolitis; PPF, postinflammatory pulmonary fibrosis 


\section{Figure 10}

Discharge Location
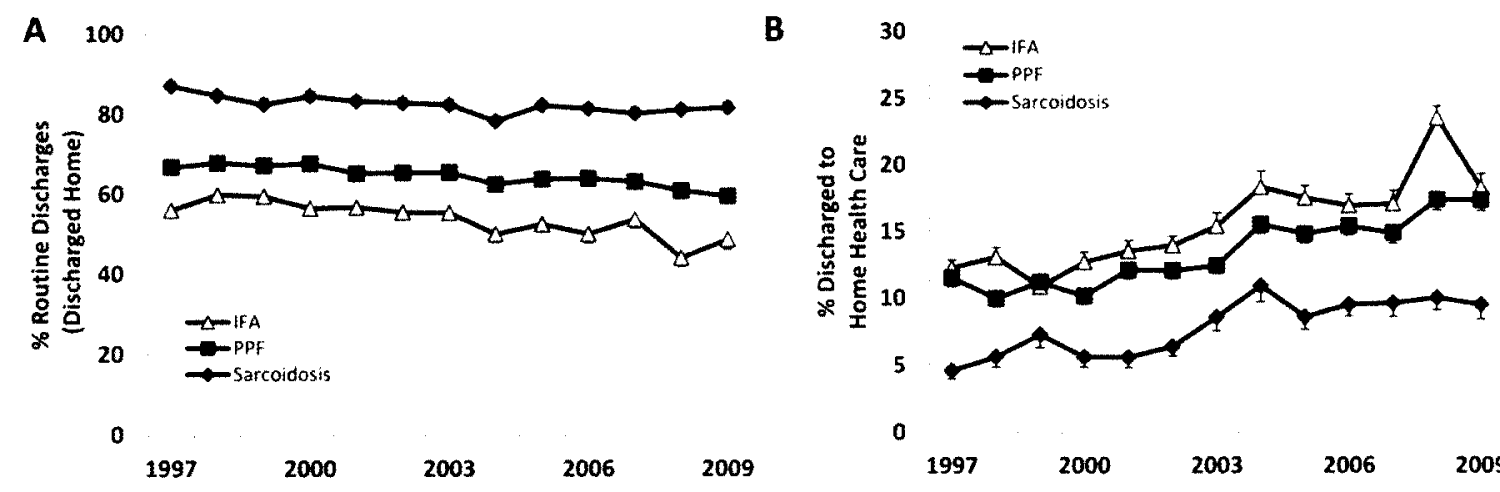

0
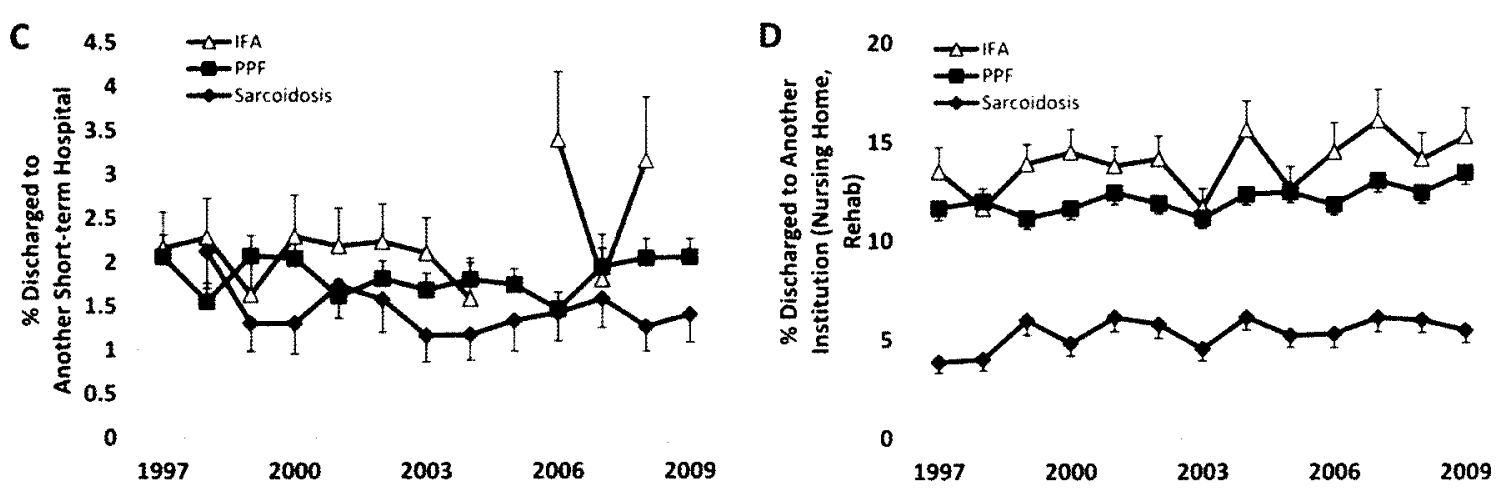

Figure $10 \mid$ A. Most patients with PPF ( $65 \%)$, IFA (50\%), and sarcoidosis $(\sim 80 \%)$ demonstrated a 'routine discharge' indicating that they returned home with routine care. However, this number decreased with time. B. Over $15 \%$ of patients hospitalized for PPF and IFA were discharged to home health care. C. The smallest percentage of discharges were sent to another short-term hospital. D. For PPF and IFA, between $10 \%$ and $15 \%$ of patients were discharged to another institution, such as a nursing home or rehabilitation center; this category has increased $1.8 \%$ for both conditions over the time period examined.

IFA, idiopathic fibrosing alveolitis; PPF, postinflammatory pulmonary fibrosis 
As expected, a higher percentage of patients with a diagnosis of sarcoidosis were given a 'routine' discharge with fewer sarcoidosis patients discharged from the hospital to home health care or another facility. In terms of patients admitted to the hospital, a small percentage (less than $\sim 5 \%$ ) were admitted from another hospital, while an increasing number (reaching $\sim 50 \%$ ) were admitted from the emergency department (not shown). This suggests that there are an increasing number of patients being hospitalized due to acute complications.

\section{IN-HOSPITAL MORTALITY}

As presented in Figure 11A, IFA had the highest in-hospital mortality through the time period examined with $14.92 \%$, followed by PPF (6.95\%), and sarcoidosis showed the lowest mortality $(1.2 \%)$. There has been a small decline in in-hospital mortality for PPF from $9 \%$ in $6.95 \%$ within the timeframe studied. In-hospital mortality also decreased slightly for IFA from $16.89 \%$ to $14.92 \%$ during the same time interval. Inhospital mortality for sarcoidosis has dropped from $2.3 \%$ to $1.2 \%$. In general, in-hospital mortality is higher for men when compared to women for all three diagnoses (Figures 11B-D). In terms of in-hospital mortality by age group there did not appear to be a clear distinction for IFA (Figure 12A). For PPF the 45-64 age group had the lowest mortality apart from 18-44; however since most patients diagnosed with PPF are older, it is possible that these were misdiagnoses. The $85+$ and $65-84$ age groups had similar overall in-hospital mortalities for PPF except for a two year time span from 2003-2004 in which the percent mortality was greater for the older age group (Figure 12B). These data 


\section{Figure 11}

In-Hospital Mortality by Disease and Gender
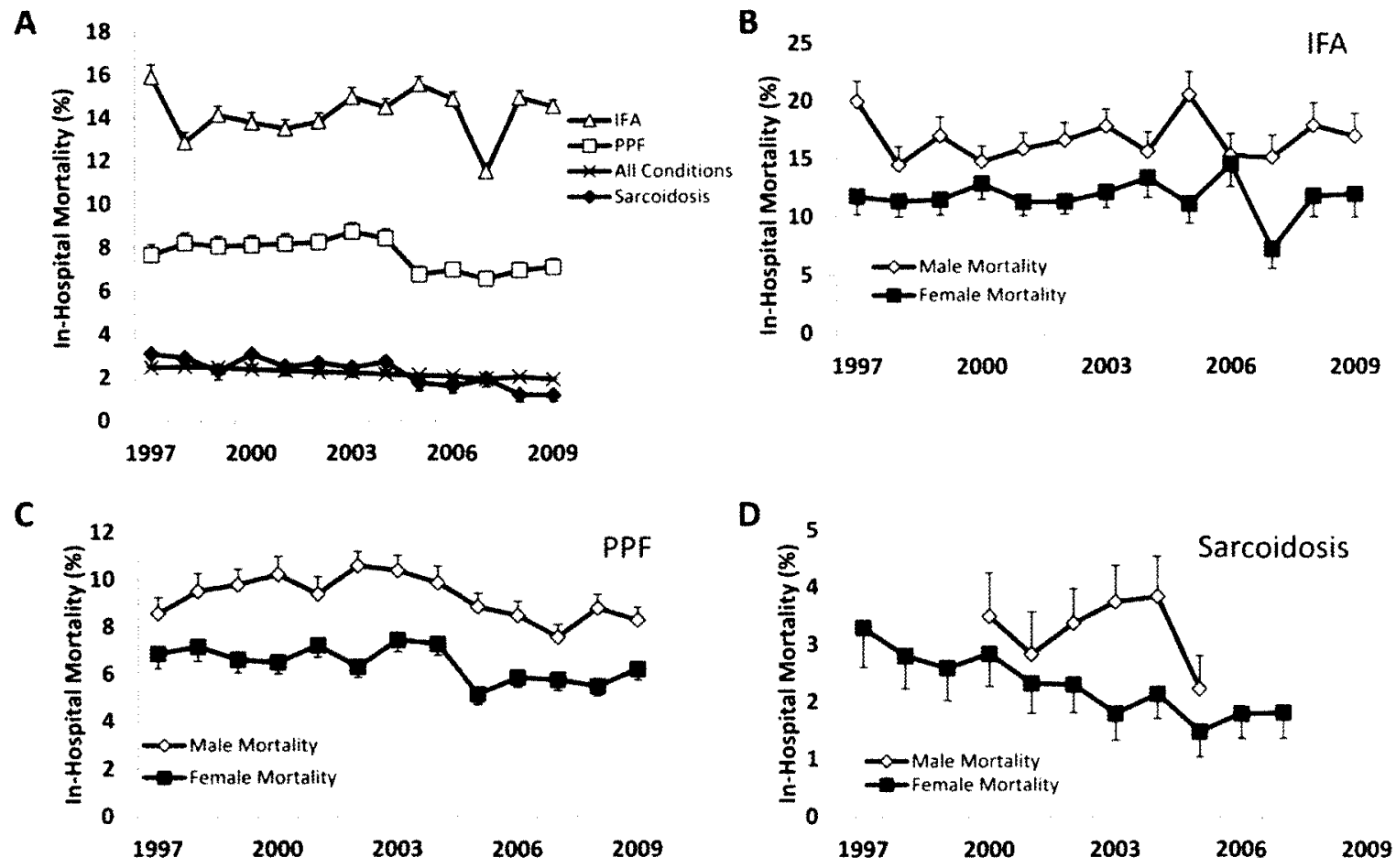

Figure 11 A. IFA had the highest in-hospital mortality through the time period examined with $14.92 \%$, followed by PPF (6.95\%), and sarcoidosis showed the lowest mortality (1.2\%). B-D. In general, in-hospital mortality is higher for men when compared to women for all three diagnoses.

IFA, idiopathic fibrosing alveolitis; PPF, postinflammatory pulmonary fibrosis 
Figure 12

$\underline{\text { In-Hospital Mortality by Age Group }}$
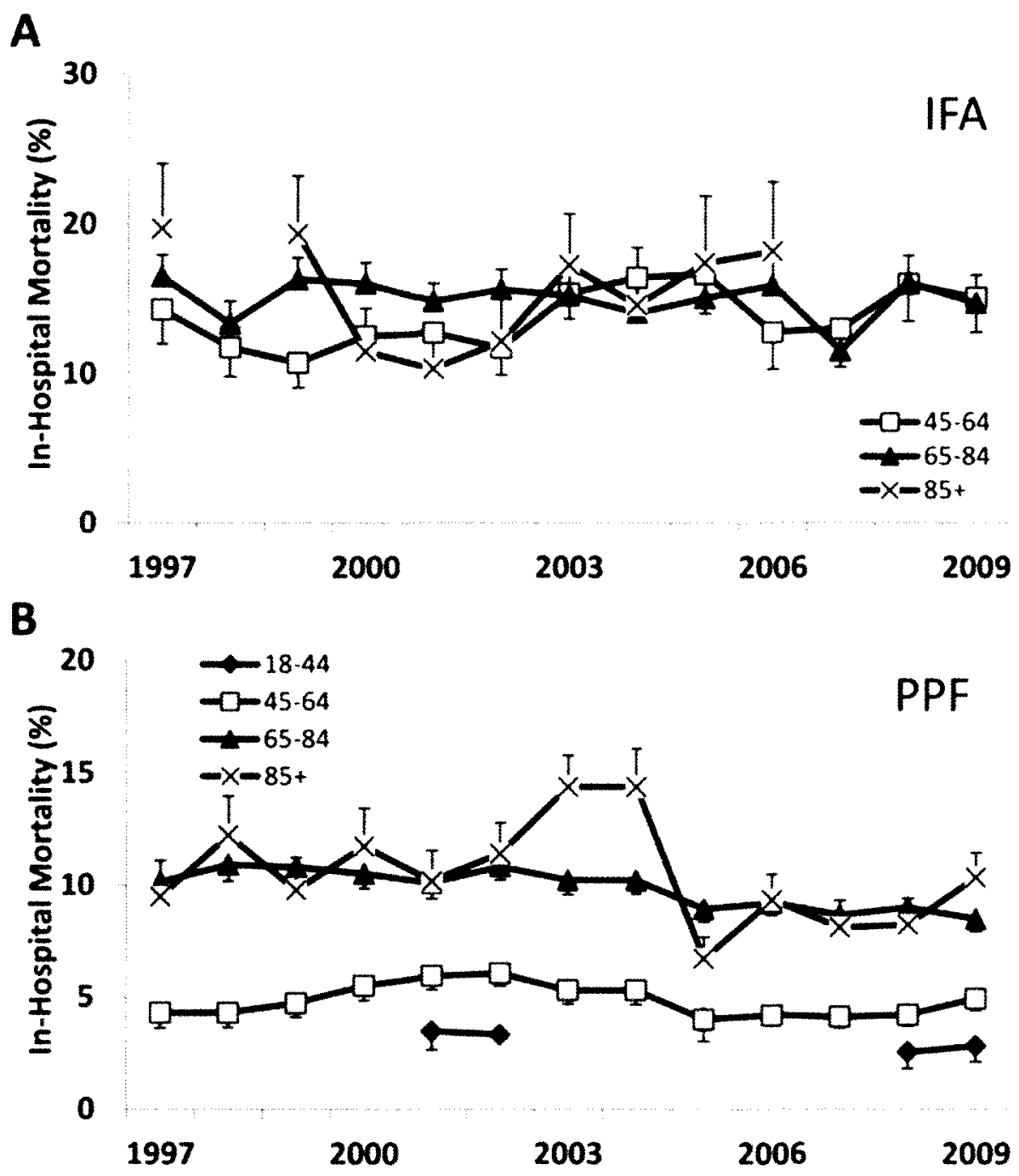

Figure $12 \mid \mathbf{A}$. In terms of in-hospital mortality by age group there did not appear to be a clear distinction for IFA. B. For PPF the 45-64 age group had the lowest mortality apart from 18-44; however since most patients diagnosed with PPF are older, it is possible that these were misdiagnoses. The $85+$ and $65-84$ age groups had similar overall in-hospital mortalities for PPF except for a two year time span from 2003-2004 in which the percent mortality was greater for the older age group.

IFA, idiopathic fibrosing alveolitis; PPF, postinflammatory pulmonary fibrosis 
suggest that after a certain age ( $\sim 65$ and older) the age of the patient does not play as important a role in affecting survival.

\section{HOSPITALIZATION CHARGES}

Hospitalization charges have increased for all conditions since 1993 reaching $\sim \$ 30,000$ on average (Figure 13A). Similarly, hospitalization charges also increased for IFA, PPF and sarcoidosis. However, while hospitalization charges for PPF $(\$ 15,000$ to over $\$ 40,000,298 \%$ increase) and sarcoidosis ( $\$ 12,563$ to over $\$ 37,000,349 \%$ increase) have increased at a similar rate, hospitalization charges for IFA have increased dramatically in recent years. IFA patients are consistently most costly, especially in the last two years of the analysis when the in-hospital charges for IFA experienced a sharp increase reaching $\$ 81,000$. Differences in hospitalization charges were noted when data were analyzed by region. For sarcoidosis and PPF, hospitalization charges were highest in the West and similar trends were noted for IFA although data for the latter diagnosis are limited (Figures 13B-D). We also analyzed mean charges by payer (Figure 14A-C). For IFA, private insurance charges were consistently one of the highest reaching over $\$ 100,000$ in the past two years. For PPF, Medicaid patients were charged the most on average from 1997-2009 and these charges were paralleled and surpassed, in the past year, by private insurance charges (which reached almost $\$ 60,000$ in 2009). Charges for uninsured patients on average were the least during the twelve year timespan. 


\section{Figure 13}

$\underline{\text { Mean Charges by Condition and Region }}$
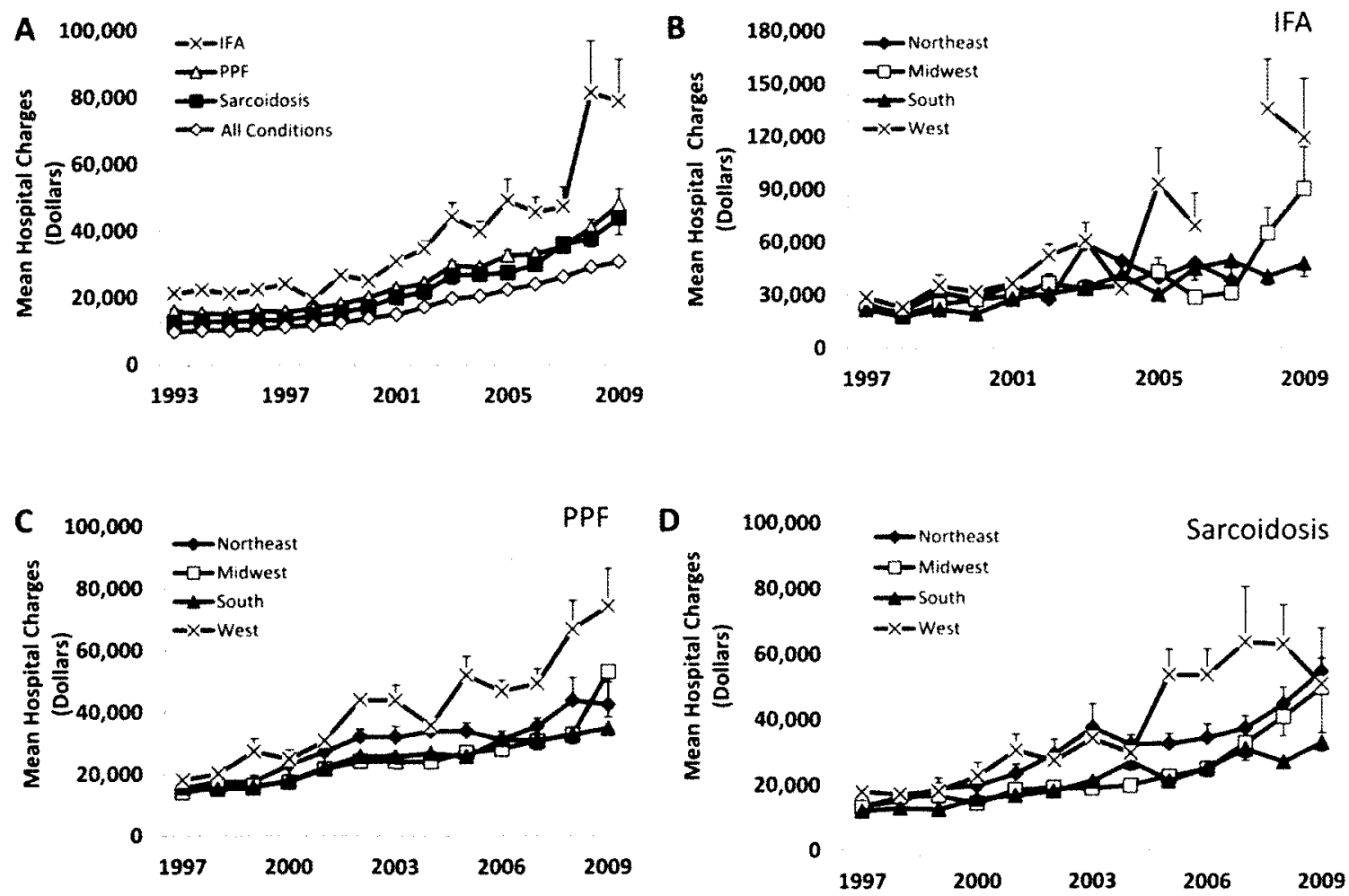

Figure 13 A. Hospitalization charges for all conditions, as well as IFA, PPF, and sarcoidosis have increased. Hospitalization charges for IFA have increased dramatically in the past two years. B-D. For sarcoidosis and PPF, hospitalization charges were highest in the West and similar trends were noted for IFA although data for the latter diagnosis are limited.

IFA, idiopathic fibrosing alveolitis; PPF, postinflammatory pulmonary fibrosis 


\section{Figure 14}

\section{Mean Charges by Payer}

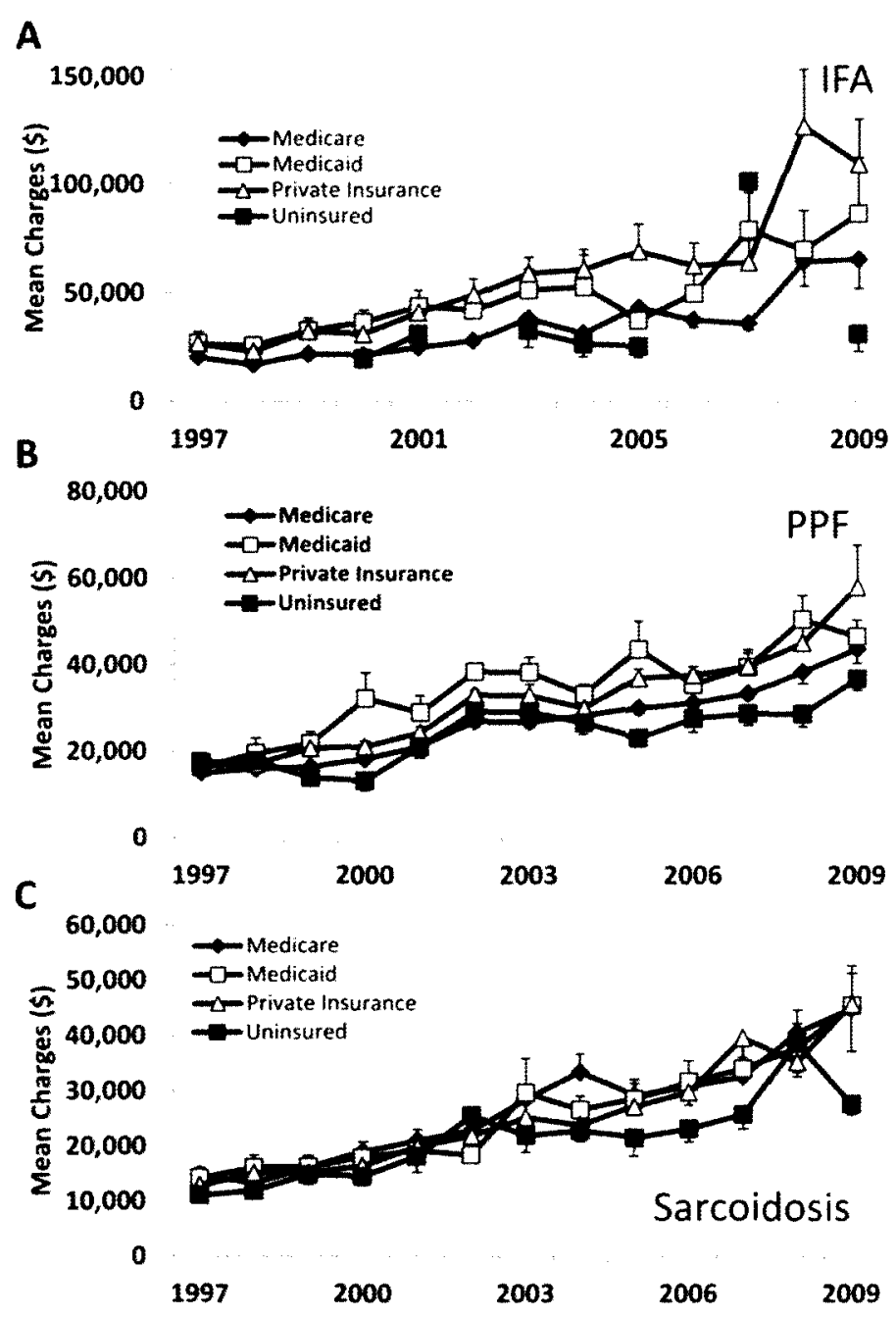

Figure $14 \mid$ A. For IFA, private insurance charges were consistently one of the highest reaching over $\$ 100,000$ in the past two years. B. For PPF, Medicaid patients were charged the most on average from 1997-2009 and these charges were paralleled and surpassed, in the past year, by private insurance charges (which reached almost $\$ 60,000$ in 2009). Charges for uninsured patients on average were the least during the twelve year timespan. C. For sarcoidosis there is no clear separation of charges by payer, except for uninsured patients for the past several years were approximately $\$ 10,000$ cheaper than other payers.

IFA, idiopathic fibrosing alveolitis; PPF, postinflammatory pulmonary fibrosis 
For sarcoidosis there is no clear separation of charges by payer, except for uninsured patients for the past several years were approximately $\$ 10,000$ cheaper than other payers. For IFA (Figure 15A) charges for males have been $\sim \$ 4,000$ higher every year and the gap has increased further in the past few years $(\sim \$ 90,000$ for males vs. $\sim \$ 60,000$ for females in 2009). Mean charges by gender were very similar for males and females for PPF and sarcoidosis (Figure 15B-C) and it is not until the past few years that a difference was seen (with males having more mean charges for both conditions). The effect of income on in-hospital mortality was analyzed (Figure 16A-B) for IFA and PPF. No distinct difference was seen for IFA and PPF suggesting that the mortality rate is not affected by the patient's income level.

Considering that there are significant differences in age of diagnosis for IFA/PPF (more common in elderly subjects) and sarcoidosis (more common in younger subjects) (Figure 17B-D), we analyzed the data for each condition based on the percent discharges based on age group and compared this to discharges for all conditions (Figure 17A). IFA and PPF had similar age distribution with the greatest percent of patients being part of the 65-84 age range (Figure 17B-C). For sarcoidosis, there was a steady increase in the percent of 45-64 year old patients, with a corresponding decrease seen in the percent of 18-44 year old patients (Figure 17D). The decrease in percent discharges of 18-44 year old patients might imply that these patients are living longer and, as such, more are surviving into older age. The decreasing mortality rate for patients with sarcoidosis also supports this idea. However the increasing average age of the American population may also be affecting these data. 


\section{Figure 15}

\section{Mean Charges by Gender}

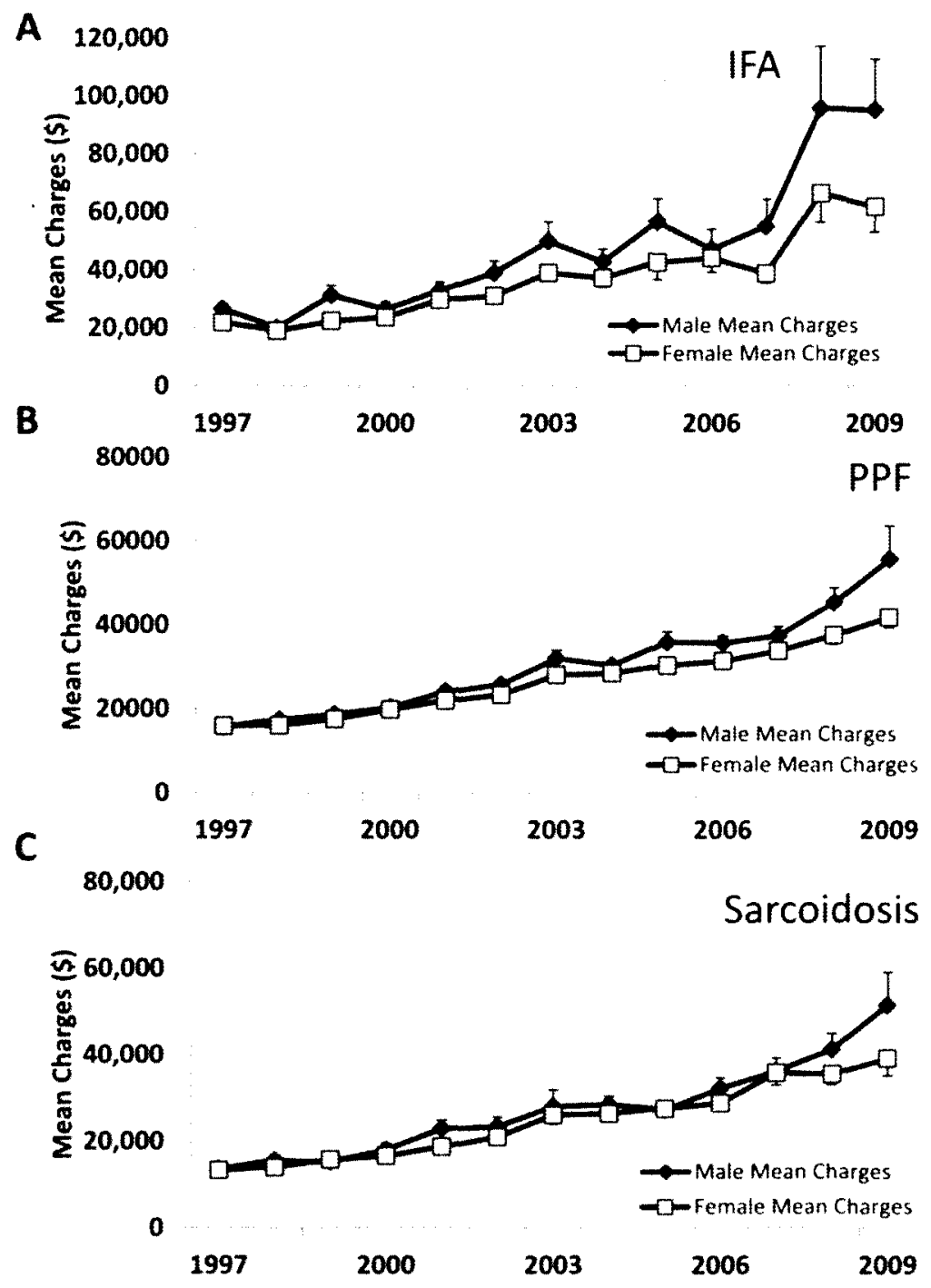

Figure $15 \mid$ A. Charges for males have been $\sim \$ 4,000$ higher every year and the gap has increased further in the past few years $(\sim \$ 90,000$ for males vs. $\sim \$ 60,000$ for females in 2009). B-C. Mean charges by gender were very similar for males and females for PPF and sarcoidosis and it is not until the past few years that a difference was seen (with males having more mean charges for both conditions).

IFA, idiopathic fibrosing alveolitis; PPF, postinflammatory pulmonary fibrosis 
Figure 16

Effect of Income on Mortality

A 25
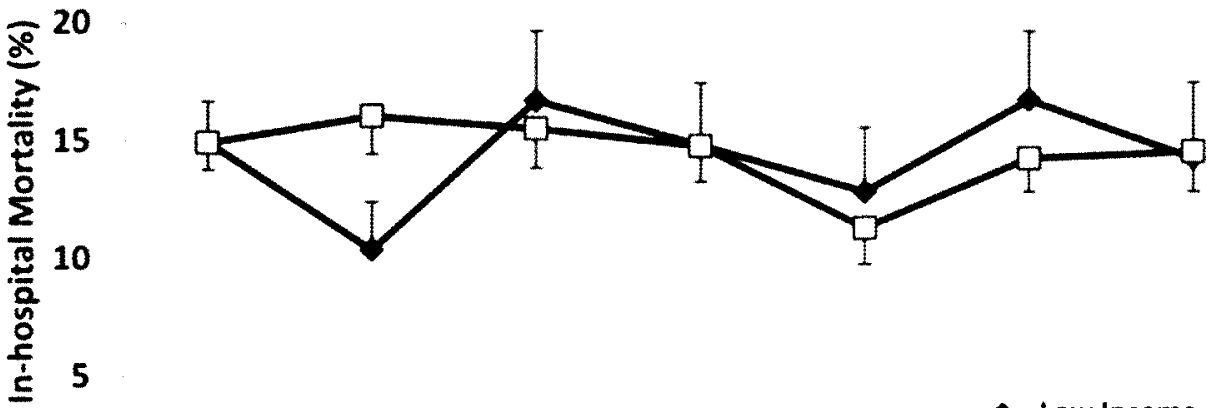

IFA

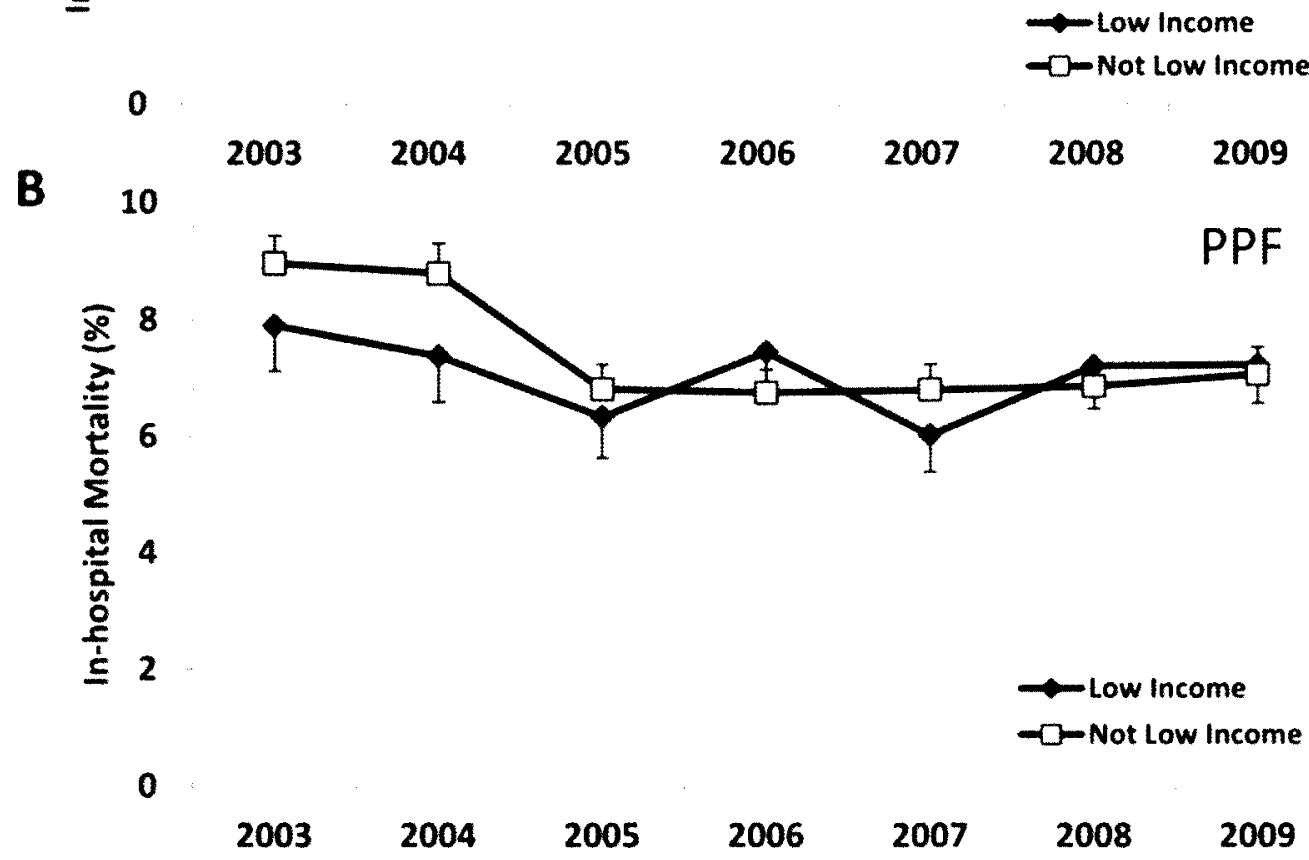

Figure $16 \mid$ A-B. The effect of income on in-hospital mortality was analyzed for IFA and PPF. No distinct difference was seen for IFA and PPF suggesting that the mortality rate is not affected by the patient's income level.

IFA, idiopathic fibrosing alveolitis; PPF, postinflammatory pulmonary fibrosis 


\section{Figure 17}

Discharges by Age Group

A

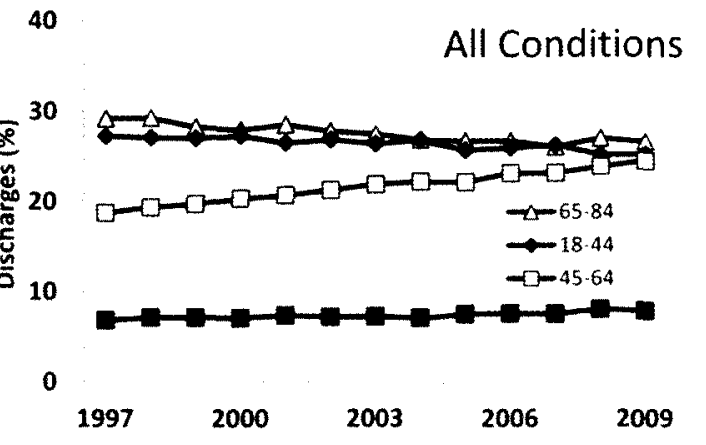

C

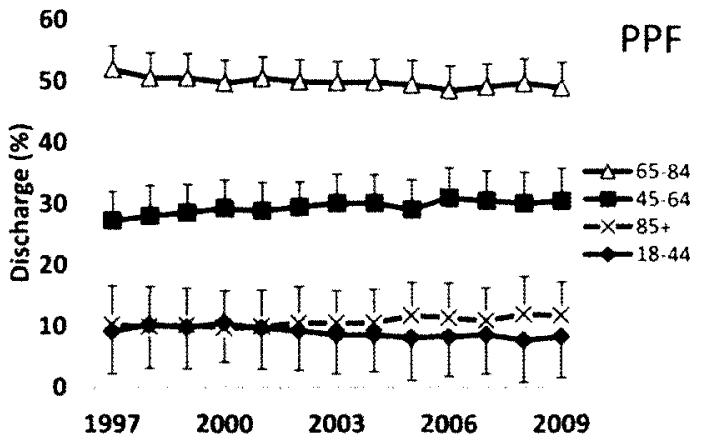

B

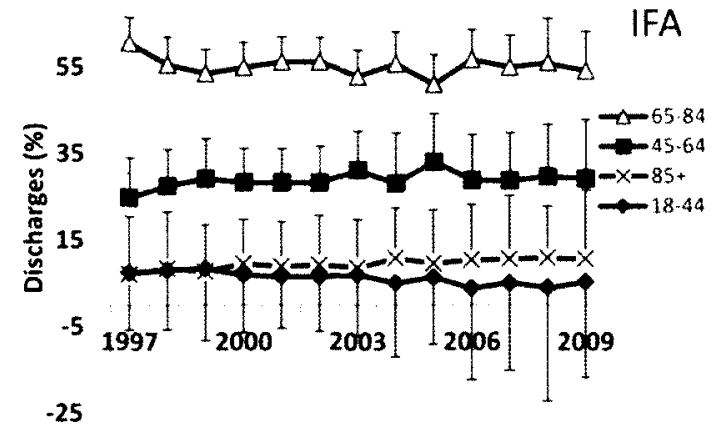

D

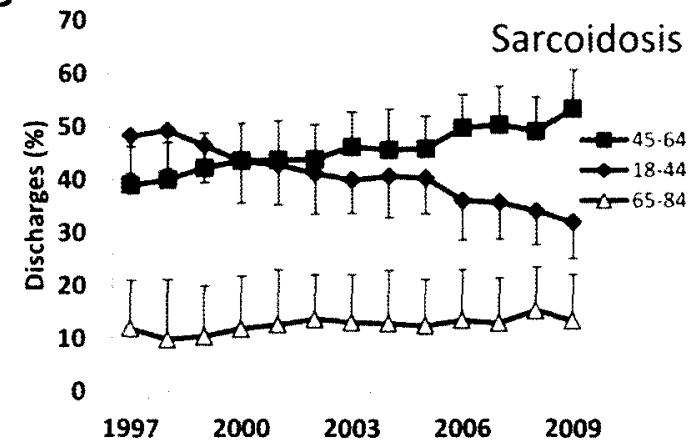

Figure $17 \mid \mathbf{A}$. Discharges for all conditions saw $\sim 5 \%$ increase in discharges for patients in the 45-64 age range. B-C. IFA and PPF had similar age distribution with the greatest percent of patients being part of the 65-84 age range. D. For sarcoidosis, there was a steady increase in the percent of 45-64 year old patients, with a corresponding decrease seen in the percent of 18-44 year old patients.

IFA, idiopathic fibrosing alveolitis; PPF, postinflammatory pulmonary fibrosis 
We further analyzed these trends by determining the number of discharges, based on condition, per million people by state for 2000 and 2010 . We arranged the states in order of youngest to oldest states (states with a smaller \% of the population older than 45 years of age were considered younger). It was hypothesized that as the average state age increased, that the number of discharges/million people would increase for IFA and PPF since these diseases are predominantly seen in elderly. However, for each entity, there was no distinct correlation between 'older' states and more discharges/million people (Figure 18A-C).

\section{DISCUSSION}

IPF is a progressive fibrotic lung disorder that affects close to 200,000 Americans and many more worldwide. Its high mortality rate, the lack of effective therapies, and recent studies showing that IPF incidence is increasing, have prompted much attention. However, little is known about IPF hospitalization rates and in-hospital mortality. It was our intention to evaluate trends for hospitalization, length of stay, in-hospital mortality, and charges for subjects carrying a diagnosis of IPF over the last two decades. However, analyzing these parameters remains a difficult task considering the limitations of the databases available and the changes that have occurred regarding the diagnosis of IPF which limit the accuracy of the classification of this disease in hospital documents for this

time period. Considering the above, we opted for evaluating hospitalizations related to the diagnosis of IFA, which is most compatible with the diagnosis of IPF, and PPF, which is likely to include all forms of pulmonary fibrosis independent of the cause. 


\section{Figure 18}

Discharges/Million People by Increasing Average State Age

A

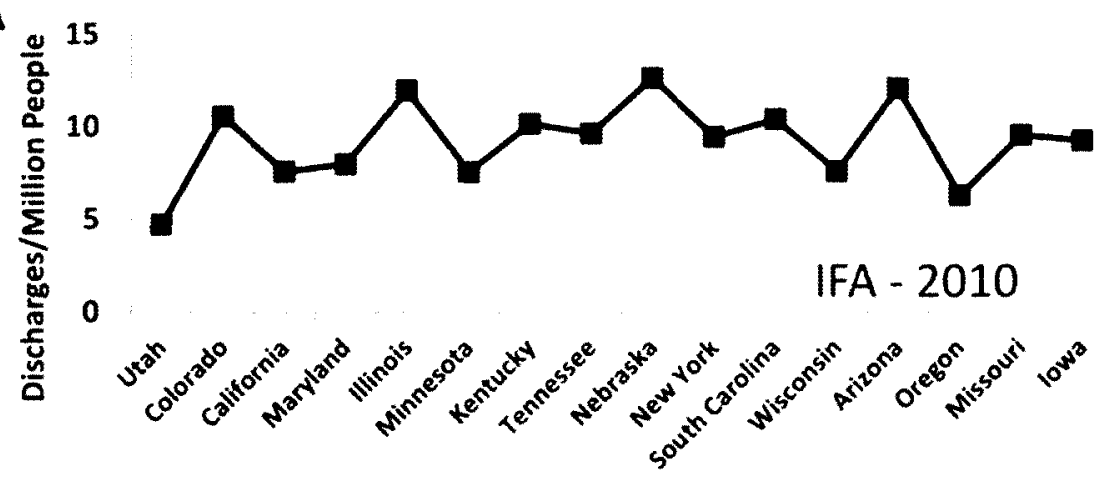

B
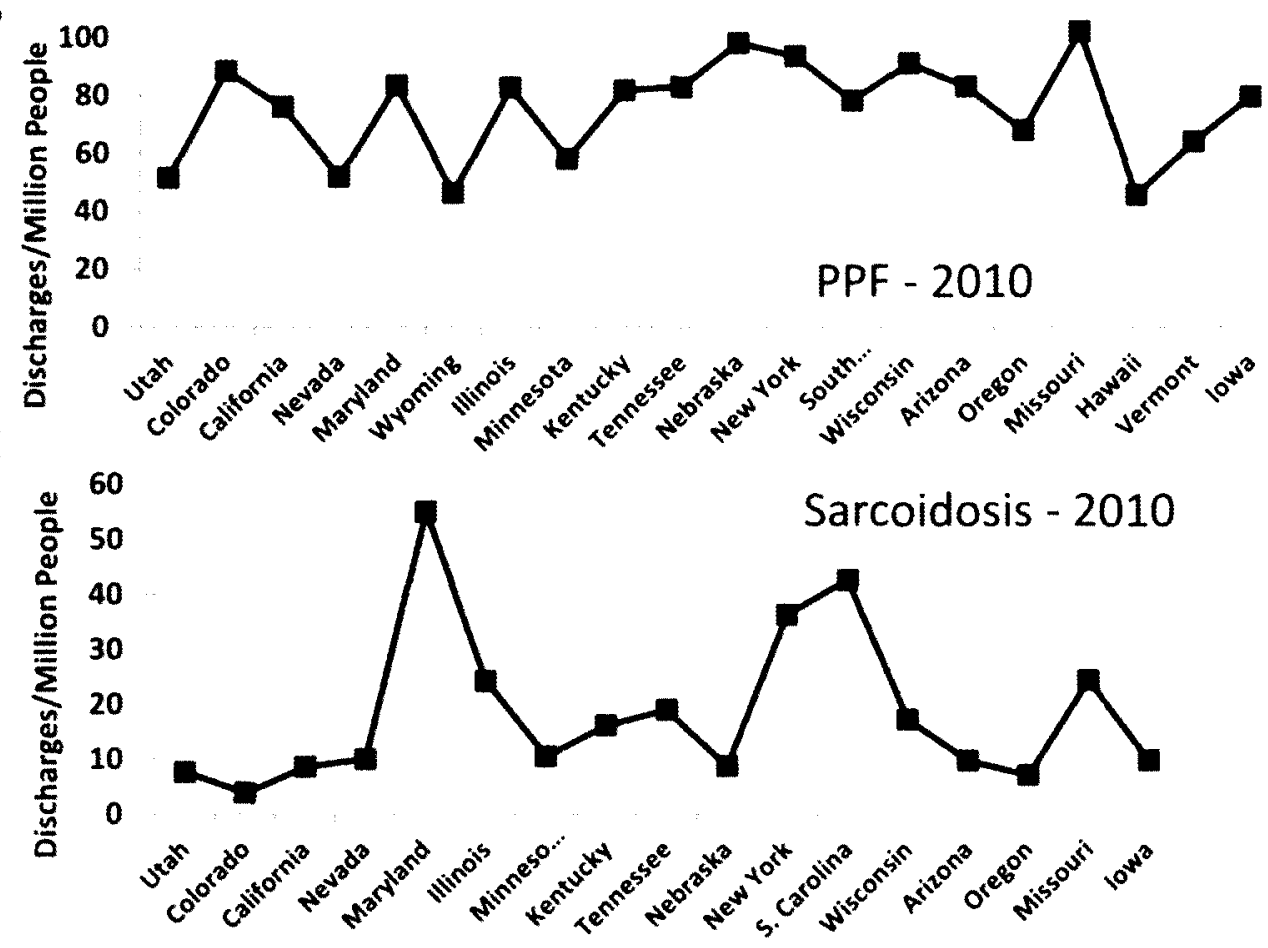

Note: States are arranged from left to right by increasing percent state population 65 years of age and older.

Figure $18 \mid$ A-C. No distinct correlation was seen between states with older populations and the number of discharges/million people.

IFA, idiopathic fibrosing alveolitis; PPF, postinflammatory pulmonary fibrosis 
Although it is likely that not all diagnoses of IFA were accurate, the database provides a window into the burden of pulmonary fibrosis in this country as it relates to hospitalization.

Analysis of these data revealed that, during the 15 year period considered by the HCUPnet Database (1993-2008), there was an increase in hospitalizations related to PPF when compared to IFA and sarcoidosis; the latter remaining relatively stable in comparison. Most patients in our cohort were hospitalized with other primary diagnoses (with PPF, IFA and sarcoidosis listed as secondary diagnoses) suggesting that subjects with these conditions are frequently hospitalized due to co-morbidities or the need for procedures. It is likely that some patients with IPF hospitalized with a diagnosis of pneumonia and congestive heart failure suffered instead from an acute exacerbation of IPF [20], but this could not be ascertained. Other co-morbidities included essential hypertension, hyperlipidemia, and diabetes; conditions typically seen in the elderly. The presence of these conditions is not surprising considering that IPF is more common with aging [3]. Secondary diagnoses related to drug toxicity were also relatively common in the IFA group, but no data were available for the other two groups. Until recently, patients with pulmonary fibrosis were frequently treated with immunosuppressive agents. The use of immunosuppressants may explain why in our cohort, up to $16 \%$ of patients hospitalized for IFA in 2008 suffered from adverse effects of medical drugs, which was associated with $18.63 \%$ in-hospital mortality.

Regarding length of stay, we found a 1-3 day higher length of stay for PPF, IFA and sarcoidosis when compared to all conditions, with IFA having the highest length of stay (7.2 days in 2008). However, over the period of the analysis, length of stay 
decreased for all three conditions. This is likely due to pressures imposed on physicians for decreasing length of stay and to the increasing recognition that many of the comorbidities related to these disorders can be treated in the ambulatory setting. No major differences were noted when gender was analyzed and when U.S. regions were compared.

We then determined where the patients were sent after their hospitalization. We noted that by 2008 , over $15 \%$ of patients hospitalized for PPF and IFA were discharged to home health care, which has been increasing steadily over the years, and a smaller percentage was discharged to another short-term hospital. For PPF and IFA, between $10 \%$ and $15 \%$ of patients were discharged to another institution, such as a nursing home or rehabilitation center. This percentage has not changed much since 1993, but certainly adds to the financial burden of families caring for loved ones with these disorders.

In-hospital mortality was highest in the IFA group (14.92\%), while PPF had $6.95 \%$ in-hospital mortality. As expected, a much lower mortality rate was noted for sarcoidosis (1.2\%). The in-hospital mortality for all groups has declined in the past years. Of note, in-hospital mortality for men was much higher than for women. This gender-based difference is quite intriguing, and some studies have reported female sex as having a protective effect and worse survival in males, but the factors responsible for it remain unclear $[64,65]$.

Finally, we examined hospitalization charges which, as expected, have increased for all conditions since 1993. Hospitalization charges have also increased for IFA, PPF and sarcoidosis. However, while hospitalization charges for PPF and sarcoidosis have increased steadily, hospitalization charges for IFA increased dramatically in recent years. 
IFA patients are consistently most costly, especially in the last two years analyzed when the in-hospital charges for IFA experienced a sharp increase reaching $\$ 81,000$. The highest charges were noted for the West region of the U.S. It is unclear how these findings are influenced by hospitalizations related to lung transplantation.

This study has a number of limitations, the most important of which relates to the accuracy of diagnosis. Considering the difficulties inherent in the diagnosis of IPF and related disorders, and the changes made in the diagnosis and classification of idiopathic interstitial pneumonias over the past two decades $[3,60]$, it is likely that there were misdiagnoses present in the database. Also, some patients may have been counted more than once since there were no unique patient identifiers [66]. However, many of the findings presented were consistent with expectations considering what we know about these disorders. For example, mortality was higher in patients with IFA and PPF when compared to sarcoidosis, while the sarcoidosis affected more females and younger individuals. The data suggest that hospitalization rates for pulmonary fibrotic disorders have increased in this country and that these disorders are associated with significant mortality and charges. When evaluating hospitalizations where the primary diagnosis was IFA or PPF, we estimate that these conditions accounted for 33,513 hospitalizations and 205,293.6 hospitalizations days in 2008. About 2,609 of these patients died while in the hospital for $7.8 \%$ in-hospital mortality. Importantly, the charges of these hospitalizations were estimated to be almost $\$ 1.5$ billion dollars in 2008 (Table 4). 


\section{Table 4}

Hospitalization charges, LOS*, and mortality for patients hospitalized for IFA*, PPF*, and sarcoidosis $(2008)^{* *}$

\begin{tabular}{llll}
\hline Parameter & IFA & PPF & Sarcoidosis \\
\hline Hospitalizations & 3,513 & 28,732 & 7,588 \\
LOS (days) & 9.3 & 6 & 6.2 \\
Mortality (\%) & 14.92 & 6.95 & 1.2 \\
Charges (\$) & 81,000 & 40,000 & 37,000 \\
$\begin{array}{l}\text { Overall charges } \\
\text { (Millions of \$) }\end{array}$ & 285 & 1,149 & 281 \\
$* * *$ Avg. Charges/ & 56,797 & 229,397 & 56,039 \\
Hospital (\$) & & & \\
*IFA, idiopathic fibrosing alveolitis; PPF, postinflammatory pulmonary fibrosis; LOS, length of stay \\
**[11], ***[67]
\end{tabular}


In conclusion, despite limitations in diagnosis accuracy, our data suggest that hospitalization rates for pulmonary fibrosis increased in the past two decades. Despite decreases in length of stay and in-hospital mortality, charges have continued to increase reaching close to 1.5 billion dollars in 2008 . The majority of these patients were admitted because of co-morbidities. Considering that we have limited options when treating fibrosis, the early identification and efficient treatment of co-morbidities might decrease hospitalizations, in-hospital mortality, and overall hospitalization charges for patients with pulmonary fibrosis. 


\section{IDENTIFYING TARGETS FOR INTERVENTION}

Understanding the impact of IPF to the healthcare system and patients is an integral step in taking measures to address the burden of this disease. However, any potential cure or drug therapy for IPF is likely to stem directly from laboratory research. In order to address the need for further understanding of disease pathogenesis, efforts were placed to understand the role of the extracellular cysteine/cystine redox potential (Eh Cys/CySS).

Oxidant stress has been implicated in the pathogenesis of IPF. In fact, a clinical trial is being conducted by the NIH-sponsored Idiopathic Pulmonary Fibrosis Clinical Research Network to test the potential role of an anti-oxidant ( $\mathrm{N}$-acetylcysteine) in the treatment of this condition; the results of this trial will not be available until late 2013 [29]. One form of oxidant stress is through oxidation of the Eh Cys/CySS, which reflects the predominant low-molecular-weight thiol/disulfide pool in the plasma [68]. Eh Cys/CySS has been found to be approximately $-80 \mathrm{mV}$ in normal individuals. However, the Eh Cys/CySS becomes more oxidized during aging and disease (e.g., cardiovascular and pulmonary diseases) as well as the exposure to chemotherapy and agents like tobacco smoke and alcohol [69].

Roman and colleagues have reported that lung fibroblasts exposed to oxidized Eh $\mathrm{Cys} / \mathrm{CySS}$ increase their proliferation as well as their expression of markers for myofibroblast transdifferentiation (i.e., $\alpha$-smooth muscle actin, fibronectin) [70]. 
Furthermore, it appears that these changes are mediated by their expression of the pro-fibrotic growth factor Transforming Growth Factor $\beta$. More recently, the Roman group has obtained plasma samples from IPF patients recruited to the IPF trial described above and found that most subjects with IPF show oxidation of their Eh Cys/CySS (unpublished results). It is for this reason that the Roman laboratory is exploring how this form of oxidant stress can affect fibroblast functions.

Considering the connection between an oxidized Eh Cys/CySS and lung fibroblasts, we turned our attention to oxidant stress and its relationship to lung fibrosis and lung cancer. Others have reported that IPF subjects have a higher incidence of lung cancer compared to controls. Based on the information presented before, we hypothesized that oxidation of the Eh Cys/CySS in IPF subjects may stimulate lung fibroblasts to secrete agents that could influence the proliferation of cancer cells. This hypothesis was tested in vitro as described below.

\section{METHODS}

\section{CELL CULTURE}

We used primary lung fibroblasts (PLF) harvested from mouse lungs as described previously [71]. Primary murine fibroblasts were obtained from C57BL/6 mice. Lung tissue sections were isolated, washed twice with sterile PBS, resuspended in DMEM supplemented with $10 \%$ FBS and $1 \%$ antibiotic-antimycotic solution $(100 \mathrm{U} / \mathrm{ml}$ penicillin G sodium, $100 \mathrm{U} / \mathrm{ml}$ streptomycin, and $0.25 \mu \mathrm{g} / \mathrm{ml}$ amphotericin $\mathrm{B}$ ), and then transferred to a tissue culture dish and incubated in a humidified $5 \% \mathrm{CO}_{2}$ incubator at $37^{\circ} \mathrm{C}$ for $1-3$ 
weeks to allow for the fibroblasts to migrate out of the tissue sections. Cells used for experiments were between passage 3 and 8 .

\section{CREATION OF CONDITIONED MEDIA}

To create the media used to treat the cancer cell lines tested (Lewis Lung Carcinoma, H1792 and H460), PLFs were plated ( $1 \times 10^{6}$ cells/well in a 6 well plate) and then grown in serum free media for 24 hours. This media was aspirated and the wells were gently washed with PBS. Redox media (of varying $\mathrm{mV}$ potentials) was then added to each of the wells of the plate (described below). Values of $-46 \mathrm{mV}$ and $-80 \mathrm{mV}$ were chosen for comparison between an oxidized and healthy state, respectively. After incubating for 24 or 72 hours in either -46 or $-80 \mathrm{mV}$ redox media, the supernatant was removed and saved. At this point, the supernatant is referred to as 'conditioned media.'

The conditioned media from the PLFs was then used for experiments with Lewis Lung Carcinoma (LLC) cells, which are lung cancer cells harvested from mice, and later, with two different human lung cancer cell lines (H460 and H1792). The respective cancer cell lines were plated in 96 well plates, grown in complete serum-free/low-protein media, and then treated with conditioned media for 24,48 , and 72 hours. After each of these time points the cells were tested for differences in proliferation using an ATP proliferation assay and apoptosis using a caspase $3 / 7$ assay.

\section{ATP PROLIFERATION AND CASPASE APOPTOSIS ASSAYS}


The ATP assay was performed using the protocol defined by Promega for their CellTiter-Glo ${ }^{\circledR}$ Luminescent Cell Viability Assay product. Briefly, the assay reagent and cell culture plate were allowed to equilibrate to room temperature. One hundred microliters of reagent were added per well in the 96-well plate and then the plate was mixed gently for 2 minutes to induce lysis. The plate was then allowed to equilibrate to room temperature for 10 minutes before luminescence (produced by the luciferase reaction) was recorded using a luminometer. The assay reagent generates a signal proportional to the amount of ATP present.

The Caspase- $3 / 7$ assay was performed according to the protocol defined by Promega for their Caspase-Glo ${ }^{\circledR} \quad 3 / 7$ Assay as described on their website (www.promega.com). Briefly, the caspase reagent and cell culture plate were brought to room temperature, 100 microliters of the reagent was added to each well of a 96 well plate. The plate was allowed to gently mix for 30 minutes before the luminescence of each sample was detected using a luminometer.

\section{ESTABLISHING EXTRACELLULAR REDOX MEDIA}

The Eh Cys/CySS was manipulated by varying concentrations of cysteine and cystine added to cyst(e)ine-free DMEM, which contains $4 \mathrm{mM}$ glutamine, $10 \mathrm{U} / \mathrm{ml}$ penicillin, and $10 \mu \mathrm{g} / \mathrm{ml}$ streptomycin.

The first step in preparation of Eh Cys/CySS involved creating $10 \mathrm{mM}$ stock solutions of cysteine and cystine, respectively. The stock solutions were adjusted to a $\mathrm{pH}$ 
of 7.4 and then sterile filtered. The stock solutions were then added in a precise ratio to cystine-free media to create -46 or $-80 \mathrm{mV}$ redox media.

\section{CALCULATION OF EH}

$$
\left.\left.E_{h}=E_{0}+R T / 2 F 1 n \text { ([cystine }\right] /[\text { cysteine }]\right)^{2}
$$

$\mathrm{E}_{\mathrm{h}}$ represents the electromotive force (given in $\mathrm{V}$ or $\mathrm{mV}$ ) relative to a standard hydrogen electrode, $\mathrm{R}$ is the gas constant, $\mathrm{T}$ is the absolute temperature, $\mathrm{F}$ is Faraday's constant, and Eo is the standard electrode potential $(-0.250 \mathrm{~V}$ at $\mathrm{pH} 7.4)$ for the cysteine/cystine redox couple.

Prior to placing the redox media on the cell line to be tested, the cells were starved in complete serum free media for 24 hours. The cells were then washed gently in PBS, the PBS was aspirated and then the cells were cultured in the -46 or $-80 \mathrm{mV}$ redox media for 24 hours.

\section{RESULTS}

First, we tested the effects of normal $(-80 \mathrm{mM})$ and oxidized $(-46 \mathrm{mM}) \mathrm{Eh}$ Cys/CySS media on the proliferation of LLCs. As presented in Figure 19, we found no differences in cell proliferation when the cells were treated directly with -46 or $-80 \mathrm{mV}$ redox media at 24, 48 and 72 hours. We, therefore, turned our attention to the effects of fibroblast conditioned media on the proliferation of LLCs. We tested the proliferation of LLCs cultured with the supernatant derived from lung fibroblasts exposed to normal and oxidized Eh Cys/CySS. Specifically, we cultured the fibroblasts in the normal and oxidized Eh Cys/CySS media for 24 or 72 hours. The proliferation of LLCs was then 
Figure 19

Lewis Lung Carcinoma (LLC) Cell Viability Assay - Response to Redox Media

A

24 Hour Cell Viability Assay - LLCs
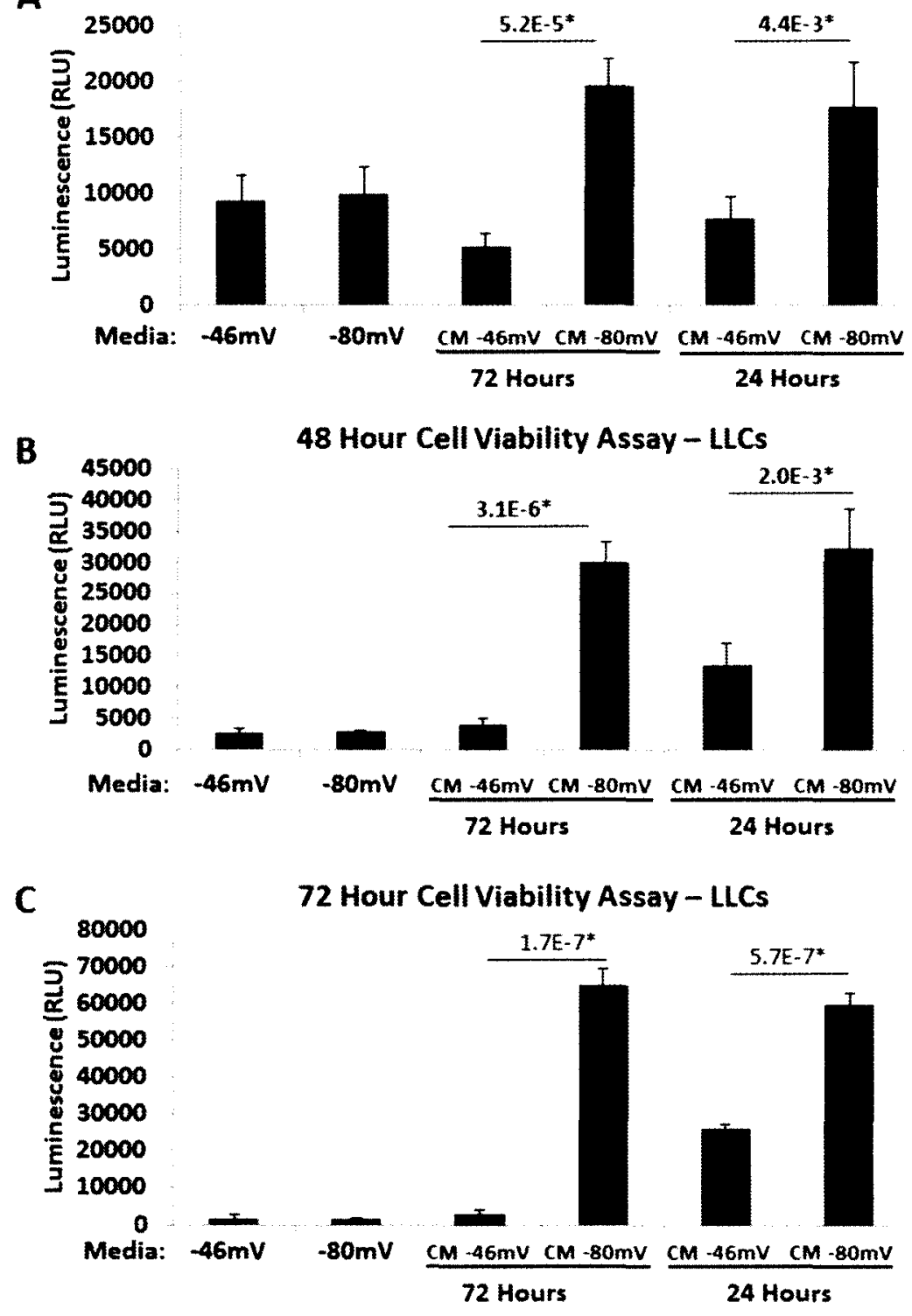

Figure 19 |A-C. No difference was seen in cell proliferation when LLCs were treated directly with -46 or $-80 \mathrm{mV}$ redox media. LLCs cultured in the presence of conditioned media (CM -46 or $-80 \mathrm{mV}$ ) showed a significant decrease in proliferation in the $\mathrm{CM}$ $-46 \mathrm{mV}$ compared to the $\mathrm{CM}-80 \mathrm{mV}$ control group. This effect was seen in response to conditioned media that was left on primary lung fibroblasts for 72 and 24 hours.

Note: $\mathrm{CM}=$ Conditioned Media (supernatant from $-46 \mathrm{mV}$ or $-80 \mathrm{mV}$ treated primary lung fibroblast cells). $\mathrm{N}=5$ for each treatment group. Error bars are standard deviations. Statistical significance was calculated using a ttest, where $\mathrm{p}<0.05$ was considered statistically significant. 
evaluated for 24, 48, and $72 \mathrm{~h}$. As seen in Figure 19, LLC cells cultured with the supernatant of lung fibroblasts exposed to oxidized Eh Cys/CySS $(-46 \mathrm{mV})$ showed decreased proliferation compared to LLCs cultured in media obtained from fibroblasts exposed to a more reduced Eh Cys/CySS $(-80 \mathrm{mV})$. This relationship was more prominent when the fibroblasts were maintained in culture for 72 hours, rather than only 24.

We then tested the effects of these manipulations on human lung cancer cells H460 and H1792. Again, we noted that direct exposure of these cells to normal and oxidized Eh Cys/CySS had no effect. However, tumor cells exposed to supernatant obtained from fibroblasts exposed to oxidized Eh Cys/CySS (indirect exposure) showed decreased proliferation when compared to LLC cells exposed to media from fibroblasts exposed to normal Eh Cys/CySS (Figures 20-21). 


\section{Figure 20}

\section{H460 and H1792 (Human Cancer Cell Lines)}

\section{Cell Viability Assay - Response to Redox Media}

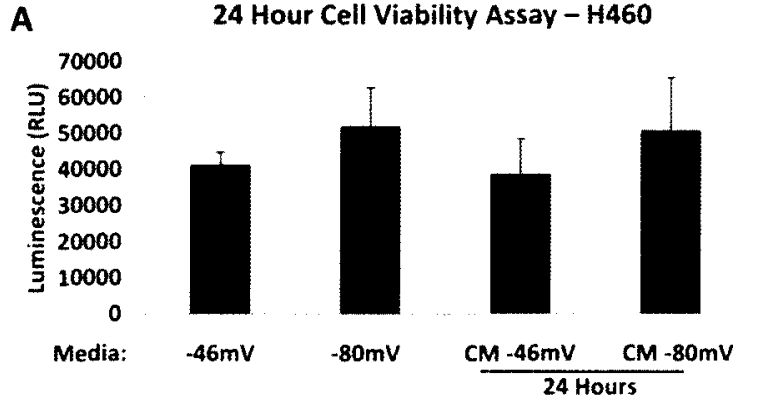

C

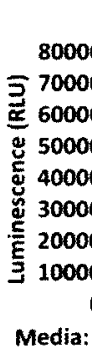

48 Hour Cell Viability Assay - 4460

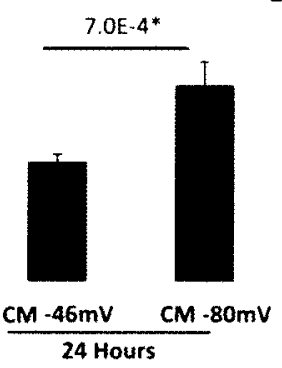

B

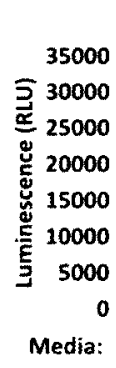

24 Hour Cell Viability Assay - $\mathbf{H 1 7 9 2}$

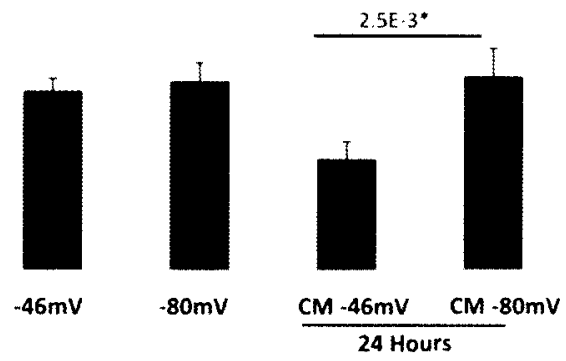

D

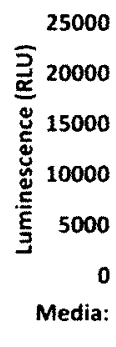

48 Hour Cell Viability Assay - H1792

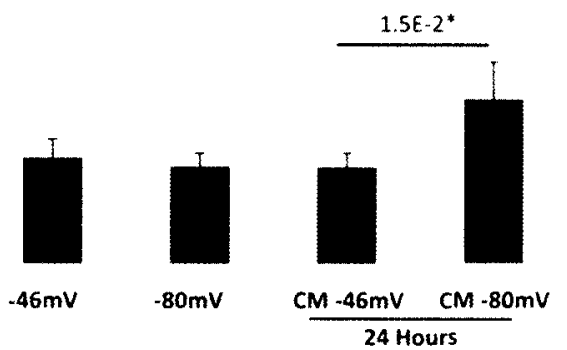

Figure 20 | A-D. No difference was seen in cell proliferation when $\mathrm{H} 460$ or $\mathrm{H} 1792$ cells were treated directly with -46 or $-80 \mathrm{mV}$ redox media. Both cell lines cultured in the presence of conditioned media $(\mathrm{CM}-46$ or $-80 \mathrm{mV})$ showed a significant decrease in proliferation in the $\mathrm{CM}-46 \mathrm{mV}$ compared to the $\mathrm{CM}-80 \mathrm{mV}$ control group. However, $\mathrm{H} 460$ cells only showed a statistically significant decrease in proliferation at 48 hours.

Note: $\mathrm{CM}=$ Conditioned Media (supernatant from $-46 \mathrm{mV}$ or $-80 \mathrm{mV}$ treated primary lung fibroblast cells). $\mathrm{N}=5$ for each treatment group. Error bars are standard deviations. Statistical significance was calculated using a ttest, where $\mathrm{p}<0.05$ was considered statistically significant. 
Figure 21

H460 and H1792 Human Cancer Cells - Response to Redox Media (Continued)

A

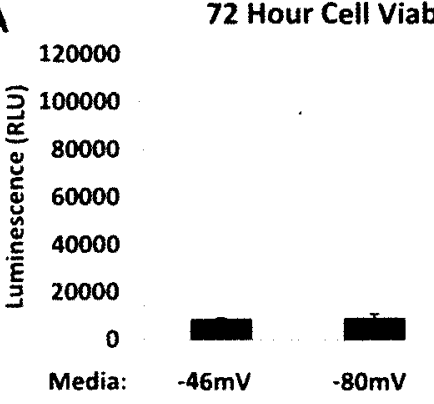

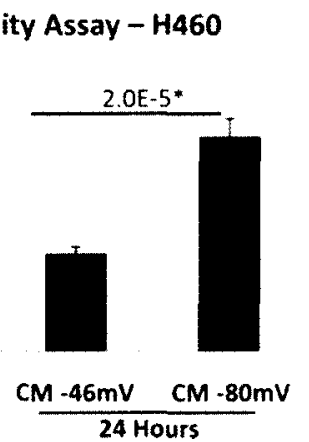

B

25000

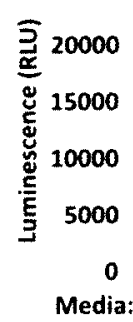

72 Hour Cell Viability Assay - $\mathbf{H 1 7 9 2}$
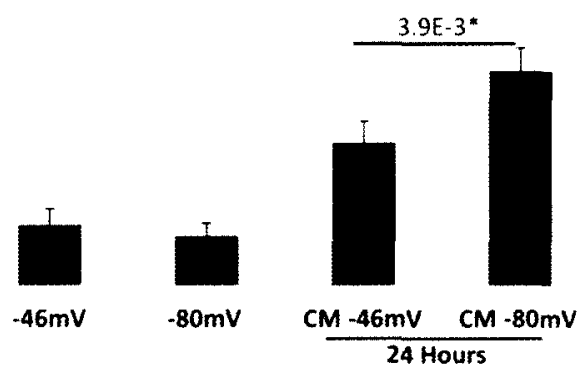

Figure 21 | A-B. No difference was seen in cell proliferation when $\mathrm{H} 460$ or $\mathrm{H} 1792$ cells were treated directly with -46 or $-80 \mathrm{mV}$ redox media at 72 hours. Both cell lines cultured in the presence of conditioned media $(\mathrm{CM}-46$ or $-80 \mathrm{mV})$ showed a significant decrease in proliferation in the $\mathrm{CM}-46 \mathrm{mV}$ compared to the $\mathrm{CM}-80 \mathrm{mV}$ control group.

Note: $\mathrm{CM}=$ Conditioned Media (supernatant from $-46 \mathrm{mV}$ or $-80 \mathrm{mV}$ treated primary lung fibroblast cells). $\mathrm{N}=5$ for each treatment group. Error bars are standard deviations. Statistical significance was calculated using a ttest, where $\mathrm{p}<0.05$ was considered statistically significant. 
The caspase assay measures the amount of cleaved caspase enzymes, specifically caspase- 3 and caspase- 7 , which are the active forms present during programmed cellular death. As depicted in Figure 22, we found that apoptosis was not different in tumor cells exposed directly to normal versus oxidized Eh Cys/CySS. However, their proliferation was decreased when exposed to the conditioned media. Although proliferation was decreased, indirect exposure to the redox media (using conditioned media) did not differentially affect apoptosis.

Together, our studies suggest that oxidation of Eh Cys/CySS in subjects with IPF may stimulate lung fibroblasts to produce agents that may indirectly influence the proliferation of lung cancer cells. Specifically, the secreted agents reduce lung cancer cell proliferation. Lung fibroblasts appear to either secrete agents, or metabolize positive factors, that affect lung cancer cell proliferation. The difference in proliferation did not appear to be affected by oxidation of the Eh Cys/CySS. Thus, we conclude that an oxidized Eh Cys/CySS stimulates lung fibroblasts to produce agents, or metabolize factors, that decrease tumor cell proliferation through effects on cell cycle rather than by inducing apoptosis. Future efforts focusing on the identification of the mechanisms responsible for the effects observed could prove beneficial in the field of IPF, since this work may unveil potential targets for intervention. 
Figure 22

\section{Caspase-3/7 Assay}

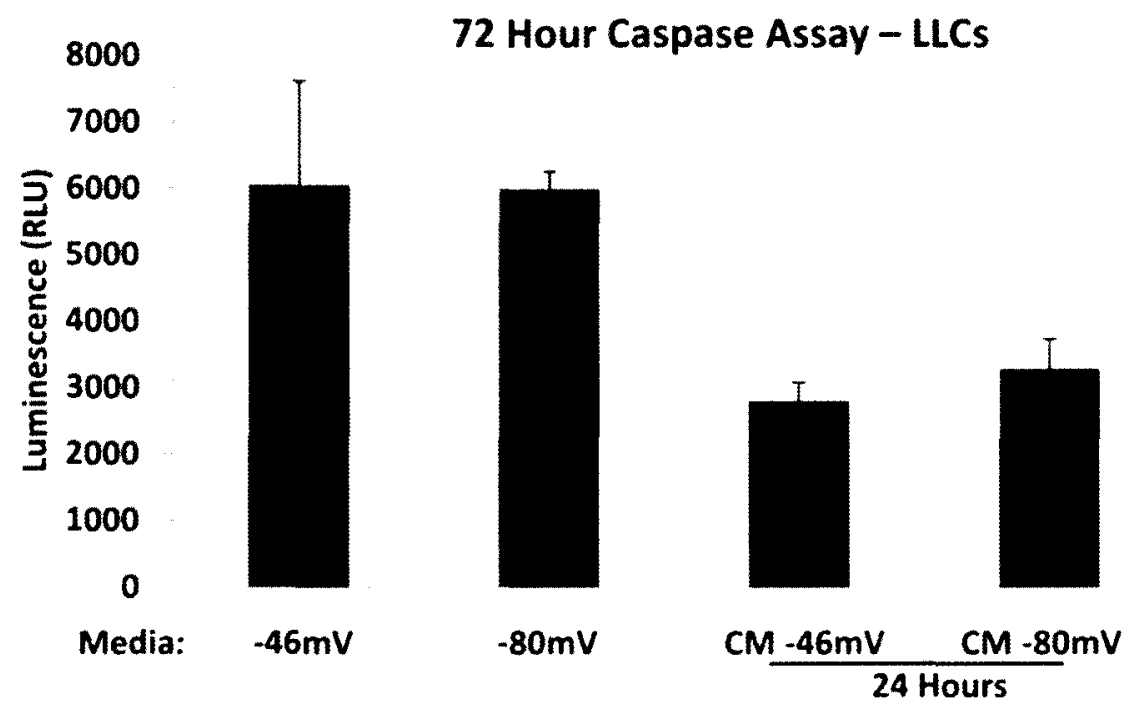

Figure 22 | Apoptosis was not different in LLCs exposed directly to normal versus oxidized Eh Cys/CySS. However, their proliferation was decreased when exposed to the conditioned media. Although proliferation was decreased, indirect exposure to the redox media (using conditioned media) did not differentially affect apoptosis.

Note: $\mathrm{CM}=$ Conditioned Media (supernatant from $-46 \mathrm{mV}$ or $-80 \mathrm{mV}$ treated primary lung fibroblast cells). LLCs $=$ Lewis Lung Carcinoma Cells. $\mathrm{N}=4$ for each treatment group. Error bars are standard deviations. Statistical significance was calculated using a ttest, where $p<0.05$ was considered statistically significant. 


\section{FUTURE WORK}

As this document suggests, efforts focused on elucidating disease pathogenesis and treatment are still greatly needed for tackling IPF as clinical trials have yet to reveal a drug to serve as a magic bullet for targeting this condition. There are several clinical trials testing new drugs/drug combination therapies, however, these trials are time sensitive and often take several years to complete and analyze. The value of these trials is great, but efforts should also be focused on determining potential genetic links to IPF. Studies of familial IPF have been essential in helping piece together a potential mechanism of disease pathogenesis, yet there is still much work that can be done in this area. Genetic studies may prove invaluable as a source for identifying genetic links to disease. Identifying genes implicated in IPF pathogenesis could provide targets for future drug therapies.

The most common animal model of IPF is the bleomycin model used in rodents. However, drug therapies shown to be effective in the bleomycin model have not been shown to provide the same clinically meaningful benefits in human studies. Alternative animal models, that better recapitulate the pattern of IPF, are needed. It is interesting to note that other species develop lung fibrosis. Lung fibrosis has been demonstrated in domesticated animals such as cats, dogs, horses and donkey as well as in birds. These animals share our environment and may represent models of naturally-occurring disease. 
If so, evaluating for clues in these models may accelerate discovery in this field, by providing a more realistic model of lung fibrosis.

Considering the ever changing healthcare landscape, it is important that we continue to evaluate the impact of IPF on healthcare costs since new information in this area may lead to alterations in the way healthcare is delivered in order to provide the best care to patients while relieving healthcare organizations and families from unnecessary financial burden. Regarding the HCUP database, we propose that further efforts should be directed towards determining a connection between states with lower mortality rates and differences in treatment/standards of care for IPF patients in that region. It needs to be determined if there are specific states with consistently lower percentages of hospital mortality. In addition, any association the lowered mortality rates may have with lower/higher hospital charges, and, most importantly, what is the cause of the lowered mortality. Factors such as local lung disease clinics (which may allow for earlier detection/treatment of IPF and any comorbidities), different drug combinations, and the quality of/access to hospitals are a few of the possibilities that should be considered.

It is clear that significant advances in the management of IPF will come only through rigorous exploration of the basic mechanisms of action responsible for the development and progression of IPF. Also, further understanding of the impact this disease has on the development of comorbidities such as lung cancer is important. The conditioned media model presented here may be used to identify fibroblast-derived products that affect lung cancer cell proliferation. 


\section{CLOSING SUMMARY}

IPF is a chronic progressive fibrotic lung disease. This disease primarily affects the elderly and has a median survival of approximately 3 years. There are no drugs that have been shown to halt or reverse the progression of this disease and lung transplantation is the only approved surgical treatment to extend survival for these patients. Patients with IPF are burdened with declining respiratory function, hospital charges that far exceed that of most conditions, and are often afflicted with comorbidities that further the difficulty of living with the disease.

Although the current situation for these patients is dire, there is promise in future efforts to treat IPF. Emerging clinical trials, increased awareness and research efforts, and a greater understanding of disease pathogenesis all point towards improved survivability, quality of life, and treatment options for IPF patients in the future. 


\section{REFERENCES}

1. Raghu, G., et al., Incidence and prevalence of idiopathic pulmonary fibrosis. Am J Respir Crit Care Med, 2006. 174(7): p. 810-6.

2. Nathan, S.D., et al., Long-term course and prognosis of idiopathic pulmonary fibrosis in the new millennium. Chest, 2011. 140(1): p. 221-9.

3. Raghu, G., et al., An official ATS/ERS/JRS/ALAT statement: idiopathic pulmonary fibrosis: evidence-based guidelines for diagnosis and management. Am J Respir Crit Care Med, 2011. 183(6): p. 788-824.

4. Nicholson, A.G., Classification of idiopathic interstitial pneumonias: making sense of the alphabet soup. Histopathology, 2002. 41(5): p. 381-91.

5. du Bois, R.M., An earlier and more confident diagnosis of idiopathic pulmonary fibrosis. Eur Respir Rev, 2012. 21(124): p. 141-6.

6. Castelino, F.V. and J. Varga, Interstitial lung disease in connective tissue diseases: evolving concepts of pathogenesis and management. Arthritis Res Ther, 2010. 12(4): p. 213.

7. Selman, M., et al., Gene expression profiles distinguish idiopathic pulmonary fibrosis from hypersensitivity pneumonitis. Am J Respir Crit Care Med, 2006. 173(2): p. 188-98.

8. Raghu, G., Improving the standard of care for patients with idiopathic pulmonary fibrosis requires participation in clinical trials. Chest, 2009. 136(2): p. 330-3.

9. Fioret, D., et al., A case of progressive lung fibrosis. Am J Med Sci, 2011. 341(6): p. 42830.

10. Fioret, D., R.L. Perez, and J. Roman, Management of idiopathic pulmonary fibrosis. Am J Med Sci, 2011. 341(6): p. 450-3.

11. Agency for Healthcare Research and Quality, Rockville, MD. November 2009; Available from: www.hcup-us.ahrq.gov/overview.jsp.

12. American Thoracic Society/European Respiratory Society International Multidisciplinary Consensus Classification of the Idiopathic Interstitial Pneumonias. This joint statement of the American Thoracic Society (ATS), and the European Respiratory Society (ERS) was adopted by the ATS board of directors, June 2001 and by the ERS Executive Committee, June 2001. Am J Respir Crit Care Med, 2002. 165(2): p. 277-304.

13. Zisman, D.A., et al., A controlled trial of sildenafil in advanced idiopathic pulmonary fibrosis. N Engl J Med, 2010. 363(7): p. 620-8.

14. Vyshedskiy, A., S. Ishikawa, and R.L. Murphy, Jr., Crackle pitch and rate do not vary significantly during a single automated-auscultation session in patients with pneumonia, congestive heart failure, or interstitial pulmonary fibrosis. Respir Care, 2011. 56(6): p. 806-17.

15. Schmidt, S.L., B. Sundaram, and K.R. Flaherty, Diagnosing fibrotic lung disease: when is high-resolution computed tomography sufficient to make a diagnosis of idiopathic pulmonary fibrosis? Respirology, 2009. 14(7): p. 934-9. 
16. Raghu, G., et al., The accuracy of the clinical diagnosis of new-onset idiopathic pulmonary fibrosis and other interstitial lung disease: A prospective study. Chest, 1999. 116(5): p. 1168-74.

17. Hunninghake, G.W., et al., Utility of a lung biopsy for the diagnosis of idiopathic pulmonary fibrosis. Am J Respir Crit Care Med, 2001. 164(2): p. 193-6.

18. Flaherty, K.R., et al., Radiological versus histological diagnosis in UIP and NSIP: survival implications. Thorax, 2003. 58(2): p. 143-8.

19. Travis, W.D., et al., Idiopathic nonspecific interstitial pneumonia: prognostic significance of cellular and fibrosing patterns: survival comparison with usual interstitial pneumonia and desquamative interstitial pneumonia. Am J Surg Pathol, 2000. 24(1): p. 19-33.

20. Collard, H.R., et al., Acute exacerbations of idiopathic pulmonary fibrosis. Am J Respir Crit Care Med, 2007. 176(7): p. 636-43.

21. Parambil, J.G., J.L. Myers, and J.H. Ryu, Histopathologic features and outcome of patients with acute exacerbation of idiopathic pulmonary fibrosis undergoing surgical lung biopsy. Chest, 2005. 128(5): p. 3310-5.

22. Nathan, S.D., P.W. Noble, and R.M. Tuder, Idiopathic pulmonary fibrosis and pulmonary hypertension: connecting the dots. Am J Respir Crit Care Med, 2007. 175(9): p. 875-80.

23. du Bois, R.M., Strategies for treating idiopathic pulmonary fibrosis. Nat Rev Drug Discov, 2010. 9(2): p. 129-40.

24. Raghu, G., et al., Treatment of idiopathic pulmonary fibrosis with etanercept: an exploratory, placebo-controlled trial. Am J Respir Crit Care Med, 2008. 178(9): p. 948-55.

25. King, T.E., Jr., et al., Effect of interferon gamma-1b on survival in patients with idiopathic pulmonary fibrosis (INSPIRE): a multicentre, randomised, placebo-controlled trial. Lancet, 2009. 374(9685): p. 222-8.

26. King, T.E., Jr., et al., BUILD-1: a randomized placebo-controlled trial of bosentan in idiopathic pulmonary fibrosis. Am J Respir Crit Care Med, 2008. 177(1): p. 75-81.

27. Daniels, C.E., et al., Imatinib treatment for idiopathic pulmonary fibrosis: Randomized placebo-controlled trial results. Am J Respir Crit Care Med, 2010. 181(6): p. 604-10.

28. Antoniou, K.M., et al., Long-term clinical effects of interferon gamma-1b and colchicine in idiopathic pulmonary fibrosis. Eur Respir J, 2006. 28(3): p. 496-504.

29. Raghu, G., et al., Prednisone, azathioprine, and $\mathrm{N}$-acety/cysteine for pulmonary fibrosis. N Engl J Med, 2012. 366(21): p. 1968-77.

30. Noth, I., et al., A Placebo-Controlled Randomized Trial of Warfarin in Idiopathic Pulmonary Fibrosis. Am J Respir Crit Care Med, 2012. 186(1): p. 88-95.

31. Raghu, G., et al., Azathioprine combined with prednisone in the treatment of idiopathic pulmonary fibrosis: a prospective double-blind, randomized, placebo-controlled clinical trial. Am Rev Respir Dis, 1991. 144(2): p. 291-6.

32. Kubo, H., et al., Anticoagulant therapy for idiopathic pulmonary fibrosis. Chest, 2005. 128(3): p. 1475-82.

33. Azuma, A., et al., Double-blind, placebo-controlled trial of pirfenidone in patients with idiopathic pulmonary fibrosis. Am J Respir Crit Care Med, 2005. 171(9): p. 1040-7.

34. Tomioka, H., et al., A pilot study of aerosolized $\mathrm{N}$-acetylcysteine for idiopathic pulmonary fibrosis. Respirology, 2005. 10(4): p. 449-55.

35. Noble, P.W., et al., Pirfenidone in patients with idiopathic pulmonary fibrosis (CAPACITY): two randomised trials. Lancet, 2011. 377(9779): p. 1760-9.

36. Ziesche, R., et al., A preliminary study of long-term treatment with interferon gamma-1b and low-dose prednisolone in patients with idiopathic pulmonary fibrosis. N Engl J Med, 1999. 341(17): p. 1264-9. 
37. Demedts, M., et al., High-dose acety/cysteine in idiopathic pulmonary fibrosis. N Engl J Med, 2005. 353(21): p. 2229-42.

38. Muller-Quernheim, J. and A. Wells, Evidence-based Recommendations in Idiopathic Pulmonary Fibrosis: A Year Is a Long Time in Interstitial Lung Disease. Am J Respir Crit Care Med, 2012. 186(1): p. 5-7.

39. Azuma, A., Pirfenidone: antifibrotic agent for idiopathic pulmonary fibrosis. Expert Rev Respir Med, 2010. 4(3): p. 301-10.

40. Grutters, J.C. and R.M. du Bois, Genetics of fibrosing lung diseases. Eur Respir J, 2005. 25(5): p. 915-27.

41. Samara, K.D., et al., Smoking and pulmonary fibrosis: novel insights. Pulm Med, 2011. 2011: p. 461439.

42. Raghu, G. and K.C. Meyer, Silent gastro-oesophageal reflux and microaspiration in IPF: mounting evidence for anti-reflux therapy? Eur Respir J, 2012. 39(2): p. 242-5.

43. Becklake, M.R., E. Bagatin, and J.A. Neder, Asbestos-related diseases of the lungs and pleura: uses, trends and management over the last century. Int J Tuberc Lung Dis, 2007. 11(4): p. 356-69.

44. Antin-Ozerkis, D., et al., Interstitial lung disease in the connective tissue diseases. Clin Chest Med, 2012. 33(1): p. 123-49.

45. Garcia-Sancho, C., et al., Familial pulmonary fibrosis is the strongest risk factor for idiopathic pulmonary fibrosis. Respir Med, 2011. 105(12): p. 1902-7.

46. Garcia, C.K., Idiopathic pulmonary fibrosis: update on genetic discoveries. Proc Am Thorac Soc, 2011. 8(2): p. 158-62.

47. Lawson, W.E., J.E. Loyd, and A.L. Degryse, Genetics in pulmonary fibrosis--familial cases provide clues to the pathogenesis of idiopathic pulmonary fibrosis. Am J Med Sci, 2011. 341(6): p. 439-43.

48. Lederer, D.J., et al., Racial and ethnic disparities in idiopathic pulmonary fibrosis: $A$ UNOS/OPTN database analysis. Am J Transplant, 2006. 6(10): p. 2436-42.

49. Lederer, D.J., et al., Racial and ethnic disparities in survival in lung transplant candidates with idiopathic pulmonary fibrosis. Am J Transplant, 2006. 6(2): p. 398-403.

50. Castriotta, R.J., et al., Workshop on idiopathic pulmonary fibrosis in older adults. Chest, 2010. 138(3): p. 693-703.

51. Ryerson, C.J., et al., Dyspnea in idiopathic pulmonary fibrosis: a systematic review. J Pain Symptom Manage, 2012. 43(4): p. 771-82.

52. Key, A.L., et al., Objective cough frequency in Idiopathic Pulmonary Fibrosis. Cough, 2010. 6: p. 4.

53. Doherty, M.J., et al., Capsaicin induced cough in cryptogenic fibrosing alveolitis. Thorax, 2000. 55(12): p. 1028-32.

54. Madison, J.M. and R.S. Irwin, Chronic cough in adults with interstitial lung disease. Curr Opin Pulm Med, 2005. 11(5): p. 412-6.

55. Hope-Gill, B.D., et al., A study of the cough reflex in idiopathic pulmonary fibrosis. Am J Respir Crit Care Med, 2003. 168(8): p. 995-1002.

56. Ley, B., H.R. Collard, and T.E. King, Jr., Clinical course and prediction of survival in idiopathic pulmonary fibrosis. Am J Respir Crit Care Med, 2011. 183(4): p. 431-40.

57. De Oliveira, N.C., et al., Lung transplant for interstitial lung disease: outcomes before and after implementation of the united network for organ sharing lung allocation scoring system. Eur J Cardiothorac Surg, 2012. 41(3): p. 680-5.

58. Lettieri, C.J., et al., Prevalence and outcomes of pulmonary arterial hypertension in advanced idiopathic pulmonary fibrosis. Chest, 2006. 129(3): p. 746-52. 
59. Lynch, D.A., et al., High-resolution computed tomography in idiopathic pulmonary fibrosis: diagnosis and prognosis. Am J Respir Crit Care Med, 2005. 172(4): p. 488-93.

60. American Thoracic Society. Idiopathic pulmonary fibrosis: diagnosis and treatment. International consensus statement. American Thoracic Society (ATS), and the European Respiratory Society (ERS). Am J Respir Crit Care Med, 2000. 161(2 Pt 1): p. 646-64.

61. Olson, A.L., et al., Seasonal variation: mortality from pulmonary fibrosis is greatest in the winter. Chest, 2009. 136(1): p. 16-22.

62. Olson, A.L., et al., Mortality from pulmonary fibrosis increased in the United States from 1992 to 2003. Am J Respir Crit Care Med, 2007. 176(3): p. 277-84.

63. Swigris, J.J., et al., Ethnic and racial differences in the presence of idiopathic pulmonary fibrosis at death. Respir Med, 2012. 106(4): p. 588-93.

64. Flaherty, K.R., et al., Clinical significance of histological classification of idiopathic interstitial pneumonia. Eur Respir J, 2002. 19(2): p. 275-83.

65. Douglas, W.W., J.H. Ryu, and D.R. Schroeder, Idiopathic pulmonary fibrosis: Impact of oxygen and colchicine, prednisone, or no therapy on survival. Am J Respir Crit Care Med, 2000. 161(4 Pt 1): p. 1172-8.

66. HCUP Frequently Asked Questions. Agency for Healthcare Research and Quality, Rockville, MD.

67. Total Hospitals. 2012; Available from: www.statehealthfacts.org.

68. Jones, D.P., Redefining oxidative stress. Antioxid Redox Signal, 2006. 8(9-10): p. 1865-79.

69. Jones, D.P., et al., Redox analysis of human plasma allows separation of pro-oxidant events of aging from decline in antioxidant defenses. Free Radic Biol Med, 2002. 33(9): p. $1290-300$.

70. Ramirez, A., et al., Extracellular cysteine/cystine redox potential controls lung fibroblast proliferation and matrix expression through upregulation of transforming growth factorbeta. Am J Physiol Lung Cell Mol Physiol, 2007. 293(4): p. L972-81.

71. Roman, J., et al., Ethanol stimulates the expression of fibronectin in lung fibroblasts via kinase-dependent signals that activate CREB. Am J Physiol Lung Cell Mol Physiol, 2005. 288(5): p. L975-87. 


\title{
CURRICULUM VITAE
}

\author{
DANIEL ROSS \\ FIORET \\ Email: \\ drfior01@louisville.edu \\ Department of Pharmacology and \\ Toxicology \\ Room 1319 Research \\ Tower \\ University of Louisville Health Sciences \\ Center \\ 500 South Preston \\ Street cell: 606-923- \\ 9503
}

\section{EDUCATION}

- 2012-2016 - Forthcoming education at the University of Louisville Medical School

- 2011-2012 - Graduate Program in Pharmacology and Toxicology

Cumulative GPA: 3.5

- 2010 - Bachelor of Arts; Spanish and Pre-Medical Focus

Cumulative GPA: 3.3

Medical College Admissions Test (MCAT): 28

Physical Sciences: 10 Biological Sciences: 10 Verbal Reasoning: 8 Centre College, Danville, KY

- 2009 - Semester Abroad in Mérida, Mexico

Semester-long study of the Spanish language, culture, and environmental biology.

- 2008 - Winter Term in Spain

Study-abroad trip in Madrid, Spain

- 2006 - Russell High School

Ashland, KY 
- 2010-Present - Pulmonary Research with Dr. Jesse Roman

- 2010-Present - Journal Club

- 2011 - Presented for Interstitial Lung Disease Weekly Meeting

\section{ABSTRACTS / PRESENTATIONS}

- 2011 - CHEST - Daniel Fioret, David Mannino MD, and Jesse Roman MD. Inhospital mortality, length of stay, and costs related to pulmonary fibrosis in the U.S. between 1993 and 2009. Presented during annual international meeting of the American College of Chest Physicians, Hawaii (October 2011)

- 2011 - IPF hospitalizations in the U.S. Interstitial Lung Disease Conference, Division of Pulmonary, Critical Care, and Sleep Medicine, Department of Medicine, University of Louisville, Louisville, KY

- 2011 - Research Louisville Poster Presentation. In-hospital mortality, length of stay, and costs related to idiopathic pulmonary fibrosis in the U.S. between 1993 and 2009

\section{PUBLICATIONS}

- Daniel Fioret, BA, Rafael Perez, MD and Jesse Roman, MD. Management of Idiopathic Pulmonary Fibrosis - June 2011 - American Journal of the Medical Sciences; Vol.341:450-453

- Daniel Fioret, BA, Rafael Perez, MD, Tamra Perez, RN and Jesse Roman, MD. A Case of Progressive Lung Fibrosis - June 2011 - American Journal of the Medical Sciences; Vol.341:428-430

- Daniel Fioret, BA, David Mannino MD, and Jesse Roman MD. In-hospital mortality, length of stay, and charges related to pulmonary fibrosis in the U.S. between 1993 and 2008 - Submitted to CHEST, awaiting response.

\section{NATIONAL / INTERNATIONAL CONFERENCES}

- CHEST 2011. Honolulu, Hawaii. Oral presentation on "In-hospital mortality, length of stay, and costs related to pulmonary fibrosis in the U.S. between 1993 and 2009." 


\section{RELEVANT COURSEWORK}

- Microbiology, Immunology, Analytical Chemistry - 2010

- Conservation Biology, Organic Chemistry I, II - 2009

- Biochemistry and Molecular Biology, General/Comparative Animal Physiology, Biology, Conservation Biology - 2008

- Accelerated General Chemistry - 2007 